\title{
Mesoscopic theory of liquid crystals ${ }^{1)}$
}

\author{
Wolfgang Muschik ${ }^{1 *}$, Christina Papenfuss ${ }^{2}$ and Harald Ehrentraut ${ }^{3}$ \\ ${ }^{1}$ Institut für Theoretische Physik, Technische Universität Berlin, Hardenbergstr. 36, \\ D-10623 Berlin, Germany (E-mail: muschik@physik.tu-berlin.de) \\ ${ }^{2}$ Institut für Mechanik, Technische Universität Berlin, Strasse des 17. Juni 135, \\ D-10623 Berlin, Germany (E-mail: cpapenfuss@c8m42.pi.tu-berlin.de) \\ ${ }^{3}$ Institut für Mechanik, Technische Universität Darmstadt, Hochschulstr. 1, \\ D-64289 Darmstadt, Germany (E-mail: harald@mechanik.tu-darmstadt.de) \\ ${ }^{*}$ Corresponding author \\ Communicated by R.F. Fox, Atlanta, USA \\ Communicated by K.-H. Hoffmann, Chemnitz, Germany
}

Registration Number 993

\begin{abstract}
Liquid crystals of uniaxial and biaxial molecules are considered in the framework of the mesoscopic description which is a general tool of continuum theory. A mesoscopic theory introduces the fields beyond hydrodynamics as additional variables of a configuration space, called mesoscopic space, on which the fields appearing in balances are defined. Besides the mesoscopic space, a mesoscopic distribution function is introduced which describes the distribution of the additional variables at each time and position. It is demonstrated, how the mesoscopic theory can be applied to liquid crystals, and how the Ericksen-Leslie theory and the alignment tensor theory of liquid crystals fit into the mesoscopic framework.
\end{abstract}

\section{Introduction}

Liquid crystals are phases showing an orientational order of molecules which are of elongated or of planar shape, so that an orientational order of them can be defined. Therefore, liquid crystals belong to the class of complex materials which need more variables for their unique description than are used in the five-field theory of fluids. Here in case of liquid crystals the orientational order has to be described by additional variables. Complex materials are wide-spread, and they represent even the

\footnotetext{
${ }^{1)}$ In memory of Frank M. Leslie. 
general case in constitutive theory. As the following examples show, the additional fields describing complex materials are of various kinds:

$$
\begin{aligned}
& \text { internal variables } \rightarrow \text { memory alloys } \\
& \text { order parameters } \rightarrow \text { phase transitions } \\
& \text { damage parameters } \rightarrow \text { fatigue problems } \\
& \text { Cosserat triads } \rightarrow \text { steel, microcrystallites, granular media } \\
& \text { conformation tensors } \rightarrow \text { polymers } \\
& \text { fabric tensors } \rightarrow \text { composite materials } \\
& \text { directors, alignment tensors } \rightarrow \text { liquid crystals. }
\end{aligned}
$$

The orientational order in liquid crystals can be differently characterized. Because of thermal fluctuations the molecules are not totally aligned, but have a certain distribution around a "mean orientation" which can be described by a normalized macroscopic director field. The name "macroscopic" originates from the fact that the macroscopic director field belongs to all molecules of a volume element, whereas a special molecule may be aligned differently. So it is obvious to introduce a microscopic director which describes the alignment of a single molecule and which is different from the local macroscopic director in general.

Besides the microscopic and the macroscopic director, other descriptions for alignment are in use which can be found in the following synopsis. Up to now there are five different phenomenological concepts suitable to describe liquid crystals nonmicroscopically. The first one is the well known Ericksen-Leslie theory $[1,2]$ whose balance equations are formulated by use of the macroscopic director mentioned above. But in fact this theory is not able to represent a change in the degree of orientational order [3]. In general we need at each point and time a distribution function for describing the macroscopic orientation of the fluid. Therefore the macroscopic director has to be redefined statistically.

The second concept describes liquid crystals as micropolar media in the frame of a 3-director theory [4]. Instead of a balance equation for the macroscopic director, the spin balance is taken into account, but no microscopic concepts are introduced.

The third concept [5] introduces besides the balance equations of a micropolar medium an additional field, called microinertia tensor field. This field, satisfying its own balance equation, is coupled to the spin balance. The form of this coupling causes all needle-shaped molecules of a volume element to always have the same angular velocity. Therefore the degree of alignment cannot change (in a co-moving frame) although it is changing from a non-zero value to zero in the phase transition from nematic to isotropic.

Consequently, the microinertia tensor field - although the molecules are not totally aligned - is not appropriate to describe dynamical situations in which the degree of alignment changes. 
The fourth concept describing liquid crystals uses the alignment-tensor [6, 7], a symmetric, traceless tensor of second order. It is defined as the first anisotropic moment of a multipole expansion of the orientation distribution function [8] (see Section 6.1). This of course is a more general method for describing alignment than using a onedirector theory.

The fifth concept describing liquid crystals introduces the above-mentioned microscopic director related to the orientation of a single rigid uniaxial molecule. The anisotropic fluid is formally treated as a mixture by regarding all particles of a volume element of the same orientation as one component of the fluid [9-13]. Thus the orientation distribution function results from the fraction of the mass density of one component by the mass density of the mixture. Because mixture theories are well developed [14, 15], balance equations for liquid crystals can be written down very easily by using this method. The domain on which these balances are defined is different from the usual one, because it contains the microscopic director as a variable. This so-called mesoscopic concept, which is discussed in more detail below, is valid for uniaxial as well as for biaxial molecules [16]. Also two-dimensional liquid crystals as films and interfaces can be treated mesoscopically [17-19].

As discussed in detail, complex materials, and among them liquid crystals, need additional fields for describing them uniquely. Now the question arises how to include these additional fields into the theory? Generally, there are two different levels of description: the microscopic level and the non-microscopic one. The microscopic level makes use of statistical physics, transport theory or of quantum theory. It is out of the scope of our considerations made here. The non-microscopic level can be split into two sub-levels, the macroscopic level and the mesoscopic one [20]. The macroscopic level is that of usual continuum theory using fields and balance equations defined on space-time. The mesoscopic level is in between both the others: it contains more information than the macroscopic description, but does not use the tools of a microscopic theory.

In the literature there is a huge flood of applications of these different theories of liquid crystals. Out of theories with a microscopic background one can distinguish socalled mean field theories, like the classical Maier-Saupe theory [21], theories taking fluctuations into account like renormalization group theories of phase transitions or theories solving the particle equations of motion by computer simulations. However, we are interested here in macroscopic theories. Examples are the macroscopic director theory of Frank, Ericksen and Leslie [2, 22-24], and the alignment tensor theory, introduced by S. Hess and Waldmann [25]. The classical Ericksen-Leslie theory deals with the field of preferred orientation in equilibrium as well as in dynamical situations in different geometries and under the action of electric and magnetic fields. Applications are for instance the optical properties and the switching behaviour of liquid crystal devices [26] or the director field in and the optical properties of liquid crystals in capillaries [27, 28] and in embedded droplets [29] (polymer dispersed liquid crystals). There exists a modification of the Ericksen-Leslie theory introducing a variable order parameter [30]. 
The aim of this paper is to present the mesoscopic theory which can describe a wide class of complex media, including liquid crystals consisting of uniaxial and biaxial molecules. The mesoscopic description of liquid crystals is compared to the EricksenLeslie director theory. In the second section we introduce the mesoscopic concept of describing liquid crystals which consists of three tools: the mesoscopic (nematic) space, the mesoscopic (orientation) distribution function and the mesoscopic (orientational) balances. The names in parentheses refer to liquid crystals. In the third section the mesoscopic balances of mass, momentum, angular momentum, etc. are written out and discussed. How to obtain back the macroscopic balances of micropolar media from the mesoscopic balances is presented in the fourth section. In the fifth section the mesoscopic distribution function and an example of orientation dynamics is discussed. The family of macroscopic order parameters which result in the alignment tensor family in the case of liquid crystals is considered in the sixth section. The Ericksen-Leslie theory of liquid crystals and liquid crystals of biaxial molecules are treated in the seventh and the ninth section. Constitutive properties described in mesoscopic theory are discussed in the eighth section. The paper closes with a summary.

\section{Mesoscopic concept}

\subsection{Mesoscopic space}

The orientation of a liquid crystal is described by one or more additional fields as discussed in the introduction. Here we choose the well defined microscopic director $\boldsymbol{n}$, describing the orientation of a single uniaxial molecule, thus having two independent components, as additional field. In general we introduce a manifold $\mathscr{M}$, called mesoscopic manifold, which is spanned by the mesoscopic variables $\boldsymbol{m} \in \mathscr{M}$ ( $\boldsymbol{m} \equiv \boldsymbol{n}$ for liquid crystals consisting of uniaxial molecules).

The conventional, macroscopic concept for including additional fields into the description is to define them on space-time $\boldsymbol{m}(\boldsymbol{x}, t): \mathbb{R}^{3} \times \mathbb{R}^{1} \rightarrow \mathscr{M}$, and to invent balances or other equations of motion which are satisfied by them. The mesoscopic concept is different and easier in the sense that fewer fields have to be defined $[20,31$. Instead of space-time, configuration spaces are introduced by the mesoscopic description. These configuration spaces are spanned by the additional variables and the space-time variables

$$
(\cdot) \equiv(\boldsymbol{m}, \boldsymbol{x}, t) \in \mathscr{M} \times \mathbb{R}^{3} \times \mathbb{R}^{1}
$$

This configuration space $(\cdot)$ is called a mesoscopic space on which now the usual fields, such as mass density $\varrho$, density of internal energy $\varepsilon$, material velocity $\boldsymbol{v}$, Cauchy stress tensor $\mathbf{T}$, heat flux density $\boldsymbol{q}, \ldots$ etc., have to be redefined. Beyond that, we have to formulate the balances of mass, momentum, energy, ... etc. on the mesoscopic space (see Section 3). This procedure is often easier than to write down balances of the additional fields defined on space-time (see Section 4). 
Because not all molecules of a volume element have the same value of the mesoscopic variable (in the case of liquid crystals the molecules have different orientation induced by thermal fluctuation for example), we have to introduce a function describing the distribution of these different values.

\subsection{Mesoscopic distribution function}

The continuum hypothesis states that a volume element is associated with each event in space-time $(\boldsymbol{x}, t)$. This hypothesis makes it possible to define macroscopic fields as mass density $\varrho(\boldsymbol{x}, t)$ and heat flux density $\boldsymbol{q}(\boldsymbol{x}, t)$. Because each volume element in space-time contains a lot of molecules having different values of the mesoscopic variables $\boldsymbol{m}$, a distribution function $f$ of these variables is associated to $(\boldsymbol{x}, t)$

$$
f(\boldsymbol{m}, \boldsymbol{x}, t) \equiv f(\cdot), \quad \int f(\boldsymbol{m}, \boldsymbol{x}, t) d \mathscr{M}=1 .
$$

The physical meaning of this so-called mesoscopic distribution function (MDF) is clear by the continuum hypothesis: in the volume element associated with $(\boldsymbol{x}, t)$ the value $\boldsymbol{m}$ of the mesoscopic variables belongs to the fraction $f$ of all molecules in the volume element. The MDF is the statistical element which characterizes the mesoscopic theory and which has no analog to the macroscopic description. This means that a mesoscopic theory contains more information than a macroscopic one. In the case of liquid crystals of uniaxial molecules, the MDF is an orientation distribution function $f(\boldsymbol{n}, \boldsymbol{x}, t)(\mathrm{ODF})$.

The MDF allows calculation of a family of macroscopic order parameters which represent additional fields in the conventional theory (see Section 6).

\subsection{Mesoscopic fields}

As the MDF $f(\cdot)$, the mesoscopic fields are defined on the mesoscopic space

$$
\boldsymbol{a}(\cdot) \equiv \boldsymbol{a}(\boldsymbol{m}, \boldsymbol{x}, t)
$$

Especially the mesoscopic mass density is defined by using the MDF

$$
\varrho(\cdot):=\varrho(\boldsymbol{x}, t) f(\cdot) .
$$

Here $\varrho(\boldsymbol{x}, t)$ is the macroscopic mass density

$$
\varrho(\boldsymbol{x}, t)=\int \varrho(\boldsymbol{m}, \boldsymbol{x}, t) d \mathscr{M}
$$

The mesoscopic mass density $\varrho(\cdot)$ describes the mass density of all molecules in a volume element for which the value of the mesoscopic variable is $\boldsymbol{m}$. In the case of liquid crystals, these are all molecules having the same orientation $\boldsymbol{n}$. 
The relation (5) is analogous to the well-known formula of mixture theory

$$
\varrho=\sum_{\alpha} \varrho^{\alpha}
$$

which states that the mass density of a mixture is the sum of the mass densities of their constituents enumerated by $\alpha$. In this diction the mesoscopic theory is a mixture theory having a continuous index describing the various species. Because mixture theory is well developed $[14,15]$, the mesoscopic theory is also easy to write out.

Other mesoscopic fields are, e.g., the mesoscopic material velocity $\boldsymbol{v}(\cdot)$, the external mesoscopic acceleration $\boldsymbol{k}(\cdot)$, the mesoscopic stress tensor $\boldsymbol{T}(\cdot)$ and the mesoscopic heat flux density $\boldsymbol{q}(\cdot)$. The meaning of these fields is clear: they all refer to the "component having the component index $\boldsymbol{m}$ ". These mesoscopic fields obey mesoscopic balance equations which are considered in the next section.

\section{Local mesoscopic balances}

Local mesoscopic balance equations can be derived from global ones. Because their local form can be understood easily, we will not deal here with the global balances which are treated in detail in [12]. From a mathematical point of view, the mesoscopic balance equations differ from the local macroscopic ones only in their domain which is enlarged by the mesoscopic variables. Therefore derivatives with respect to these variables appear in the balances

$$
\frac{\partial}{\partial t} \boldsymbol{X}(\cdot)+\nabla_{x} \cdot[\boldsymbol{v}(\cdot) \boldsymbol{X}(\cdot)-\boldsymbol{S}(\cdot)]+\nabla_{m} \cdot[\boldsymbol{u}(\cdot) \boldsymbol{X}(\cdot)-\boldsymbol{R}(\cdot)]=\Sigma(\cdot) .
$$

Here $\boldsymbol{X}(\cdot)$ is the balanced mesoscopic filed, while $\boldsymbol{S}(\cdot)$ and $\boldsymbol{R}(\cdot)$ are the conductive parts of the fluxes. The analog to the material velocity is the mesoscopic change velocity $\boldsymbol{u}(\cdot)$ which is defined as follows

$$
(\boldsymbol{m}, \boldsymbol{x}, t) \rightarrow(\boldsymbol{m}+\boldsymbol{u}(\cdot) \Delta t, \boldsymbol{x}+\boldsymbol{v}(\cdot) \Delta t, t+\Delta t),
$$

where $\boldsymbol{v}(\cdot) \boldsymbol{X}(\cdot)$ and $\boldsymbol{u}(\cdot) \boldsymbol{X}(\cdot)$ are the convective parts of the fluxes. $\Sigma(\cdot)$ is supply and production of $\boldsymbol{X}(\cdot)$. In more detail the special mesoscopic balance equations are:

\subsection{Mass}

$$
\frac{\partial}{\partial t} \varrho(\cdot)+\nabla_{x} \cdot\{\varrho(\cdot) \boldsymbol{v}(\cdot)\}+\nabla_{m} \cdot\{\varrho(\cdot) \boldsymbol{u}(\cdot)\}=0 .
$$

\subsection{Momentum}

$$
\begin{aligned}
& \frac{\partial}{\partial t}[\varrho(\cdot) \boldsymbol{v}(\cdot)]+\nabla_{x} \cdot\left[\boldsymbol{v}(\cdot) \varrho(\cdot) \boldsymbol{v}(\cdot)-\mathbf{T}^{\top}(\cdot)\right] \\
& \quad+\nabla_{m} \cdot\left[\boldsymbol{u}(\cdot) \varrho(\cdot) \boldsymbol{v}(\cdot)-\mathscr{T}^{\top}(\cdot)\right]=\varrho(\cdot) \boldsymbol{k}(\cdot) .
\end{aligned}
$$


Here $\mathscr{T}(\cdot)$ is the analog to the stress tensor, the non-convective momentum flux with respect to the mesoscopic variables $\boldsymbol{m}$.

\subsection{Angular momentum}

The angular momentum is defined by the sum

$$
\begin{aligned}
& \boldsymbol{M}(\cdot):=\boldsymbol{x} \times \boldsymbol{v}(\cdot)+\boldsymbol{s}(\cdot) \\
& \frac{\partial}{\partial t}[\varrho(\cdot) \boldsymbol{M}(\cdot)]+\nabla_{x} \cdot\left[\boldsymbol{v}(\cdot) \varrho(\cdot) \boldsymbol{M}(\cdot)-(\boldsymbol{x} \times \mathbf{T}(\cdot))^{\top}-\mathbf{W}^{\top}(\cdot)\right] \\
& \quad+\nabla_{m} \cdot\left[\boldsymbol{u}(\cdot) \varrho(\cdot) \boldsymbol{M}(\cdot)-(\boldsymbol{x} \times \mathscr{T}(\cdot))^{\top}-\mathscr{W}^{\top}(\cdot)\right] \\
& \quad=\varrho(\cdot) \boldsymbol{x} \times \boldsymbol{k}(\cdot)+\varrho(\cdot) \boldsymbol{g}(\cdot) .
\end{aligned}
$$

Here $s(\cdot)$ is the mesoscopic specific spin which occurs due to rotations of nonspherical particles, and $\boldsymbol{g}(\cdot)$ is the mesoscopic angular momentum exerted by external forces on the mesoscopic $s(\cdot)$. $W(\cdot)$ is the mesoscopic surface torque or couple stress, $\mathscr{W}(\cdot)$ the analog to $\mathbf{W}(\cdot)$ acting on the mesoscopic variables $\boldsymbol{m}$.

\subsection{Spin}

If the balance of momentum (10) is multiplied by $\times \boldsymbol{x}$ and subtracted from the balance of angular momentum (12), we obtain the spin balance

$$
\begin{aligned}
& \frac{\partial}{\partial t}[\varrho(\cdot) \boldsymbol{s}(\cdot)]+\nabla_{x} \cdot\left[\varrho(\cdot) \boldsymbol{v}(\cdot) \boldsymbol{s}(\cdot)-\mathbf{W}^{\top}(\cdot)\right] \\
& \quad+\nabla_{m} \cdot\left[\varrho(\cdot) \boldsymbol{u}(\cdot) \boldsymbol{s}(\cdot)-\mathscr{W}^{\top}(\cdot)\right]=\varepsilon: \mathbf{T}(\cdot)+\varrho(\cdot) \boldsymbol{g}(\cdot) .
\end{aligned}
$$

Here $\varepsilon$ is the Levi-Civita tensor.

\subsection{Total energy}

The total energy is defined by

$$
\begin{aligned}
& e(\cdot):=\frac{1}{2} \boldsymbol{v}^{2}(\cdot)+\frac{1}{2} \boldsymbol{s}(\cdot) \cdot \Theta^{-1} \cdot \boldsymbol{s}(\cdot)+\varepsilon(\cdot), \\
& \begin{aligned}
\frac{\partial}{\partial l}[\varrho(\cdot) e(\cdot)]+\nabla_{x} \cdot\left[\boldsymbol{v}(\cdot) \varrho(\cdot) e(\cdot)-\mathbf{T}^{\top}(\cdot) \cdot \boldsymbol{v}(\cdot)-\mathbf{W}^{\top}(\cdot) \cdot \Theta^{-1} \cdot \boldsymbol{s}(\cdot)+\boldsymbol{q}(\cdot)\right] \\
\quad+\nabla_{m} \cdot\left[\boldsymbol{u}(\cdot) \varrho(\cdot) e(\cdot)-\mathscr{T}^{\top}(\cdot) \cdot \boldsymbol{v}(\cdot)-\boldsymbol{W}^{\top}(\cdot) \cdot \Theta^{-1} \cdot \boldsymbol{s}(\cdot)+Q(\cdot)\right] \\
\quad=\varrho(\cdot) \boldsymbol{k}(\cdot) \cdot \boldsymbol{v}(\cdot)+\varrho(\cdot) \boldsymbol{g}(\cdot) \cdot \Theta^{-1} \cdot \boldsymbol{s}(\cdot)+\varrho(\cdot) r(\cdot) .
\end{aligned}
\end{aligned}
$$

Here $\varepsilon(\cdot)$ is the mesoscopic internal energy density, $r(\cdot)$ the mesoscopic absorption supply and $\Theta$ the moment of inertia tensor which couples the spin density with the 
local angular velocity. The mesoscopic heat flux density is $\boldsymbol{q}(\cdot), \boldsymbol{Q}(\cdot)$ the mesoscopic heat flux density on $\mathscr{M}$.

Taking into account the spin balance and the balance of the kinetic energy which follows from the balance of momentum we obtain the balance of internal energy.

\subsection{Internal energy}

$$
\begin{aligned}
\frac{\partial}{\partial t}[\varrho(\cdot) & \varepsilon(\cdot)]+\nabla_{x} \cdot[\varrho(\cdot) \boldsymbol{v}(\cdot) \varepsilon(\cdot)+\boldsymbol{q}(\cdot)]+\nabla_{m} \cdot[\varrho(\cdot) \boldsymbol{u}(\cdot) \varepsilon(\cdot)+\boldsymbol{Q}(\cdot)] \\
= & \varrho(\cdot) r(\cdot)-\boldsymbol{s}(\cdot) \cdot \Theta^{-1} \cdot \varepsilon: \mathbf{T}(\cdot)+\mathbf{T}^{\top}(\cdot):\left[\nabla_{x} \boldsymbol{v}(\cdot)\right] \\
& +\mathscr{T}^{\top}(\cdot):\left[\nabla_{m} \boldsymbol{v}(\cdot)\right]+\mathbf{W}^{\top}(\cdot): \nabla_{x}\left(\Theta^{-1} \cdot \boldsymbol{s}(\cdot)\right) \\
& +\mathscr{W}^{\top}(\cdot): \nabla_{m}\left(\Theta^{-1} \cdot \boldsymbol{s}(\cdot)\right) .
\end{aligned}
$$

The mesoscopic balance of entropy is of no special interest, because its production density is not definite in general. Hence we cannot use it for the exploitation of the Second Law which has to be formulated as usual by the macroscopic balance of entropy.

\subsection{Macroscopic entropy}

$$
\frac{\partial}{\partial t}[\varrho(\boldsymbol{x}, t) s(\boldsymbol{x}, t)]+\nabla_{x} \cdot[\boldsymbol{v} \varrho(\boldsymbol{x}, t) s(\boldsymbol{x}, t)+\Phi(\boldsymbol{x}, t)]-\pi(\boldsymbol{x}, t)=\sigma(\boldsymbol{x}, t) \geq 0 .
$$

Here $s(\boldsymbol{x}, t)$ is the macroscopic entropy density and $\boldsymbol{\Phi}(\boldsymbol{x}, t)$ the macroscopic entropy flux density. The entropy supply is $\pi(\boldsymbol{x}, t)$ and $\sigma(\boldsymbol{x}, t)$ the non-negative entropy production.

The mesoscopic balances do not contain any new balance for the additional fields, because those are included by introducing the mesoscopic space. The new four fields, MDF $f(\cdot)$ and mesoscopic change velocity $\boldsymbol{u}(\cdot)$, require equations for determining them. The MDF has a balance for its own (see Section 5). The mesoscopic change velocity is the only new balance we need. In special cases the mesoscopic spin balance may be sufficient to determine $\boldsymbol{u}(\cdot)$ (see Section 5.2).

Now we will rediscover the macroscopic balances from the mesoscopic ones.

\section{Macroscopic balances}

The balance equations of micropolar media can be obtained from the mesoscopic balances by replacing the mesoscopic space by space-time

$$
(\cdot) \equiv(\boldsymbol{m}, \boldsymbol{x}, t) \rightarrow(\boldsymbol{x}, t) .
$$


Because the mesoscopic variables $\boldsymbol{m}$ do not appear any more, the derivatives with respect to $\boldsymbol{m}$ have to be set to zero

$$
\nabla_{m} \cdot[\ldots] \rightarrow 0
$$

By this formal setting we formulate the following axiom.

Axiom: The mesoscopic balance (7) and the macroscopic balance equation

$$
\frac{\partial}{\partial t} \boldsymbol{X}(\boldsymbol{x}, t)+\nabla_{x} \cdot[\boldsymbol{v}(\boldsymbol{x}, t) \boldsymbol{X}(\boldsymbol{x}, t)-\boldsymbol{S}(\boldsymbol{x}, t)]=\Sigma(\boldsymbol{x}, t)
$$

are related to each other.

Using this axiom we obtain from the mesoscopic balances the following macroscopic ones:

\subsection{Macroscopic mass}

$$
\frac{\partial}{\partial t}\left[\varrho(\boldsymbol{x}, t)+\nabla_{x} \cdot\{\varrho(\boldsymbol{x}, t) \boldsymbol{v}(\boldsymbol{x}, t)\}=0 .\right.
$$

\subsection{Macroscopic momentum}

$$
\frac{\partial}{\partial t}\left[\varrho(\boldsymbol{x}, t) \boldsymbol{v}(\boldsymbol{x}, t)+\nabla_{x} \cdot\left[\boldsymbol{v}(\boldsymbol{x}, t) \varrho(\boldsymbol{x}, t) \boldsymbol{v}(\boldsymbol{x}, t)-\boldsymbol{T}^{\top}(\boldsymbol{x}, t)\right]=\varrho(\boldsymbol{x}, t) \boldsymbol{k}(\boldsymbol{x}, t) .\right.
$$

\subsection{Macroscopic angular momentum}

$$
\begin{aligned}
& \boldsymbol{M}(\boldsymbol{x}, t):=\boldsymbol{x} \times \boldsymbol{v}(\boldsymbol{x}, t)+\boldsymbol{s}(\boldsymbol{x}, t), \\
& \frac{\partial}{\partial t}[\varrho(\boldsymbol{x}, t) \boldsymbol{M}(\boldsymbol{x}, t)]+\nabla_{x} \cdot\left[\boldsymbol{v}(\boldsymbol{x}, t) \varrho(\boldsymbol{x}, t) \boldsymbol{M}(\boldsymbol{x}, t)-(\boldsymbol{x} \times \mathbf{T}(\boldsymbol{x}, t))^{\top}-\mathbf{W}^{\top}(\boldsymbol{x}, t)\right] \\
& \quad=\varrho(\boldsymbol{x}, t) \boldsymbol{x} \times \boldsymbol{k}(\boldsymbol{x}, t)+\varrho(\boldsymbol{x}, t) \boldsymbol{g}(\boldsymbol{x}, t) .
\end{aligned}
$$

Here $\boldsymbol{s}(\boldsymbol{x}, t)$ is the macroscopic specific spin and $\boldsymbol{g}(\boldsymbol{x}, t)$ the macroscopic angular momentum exerted by external forces on the spin $\boldsymbol{s}(\boldsymbol{x}, l) . \mathbf{W}(\boldsymbol{x}, l)$ is the macroscopic surface torque or couple stress.

\subsection{Macroscopic spin}

$$
\begin{gathered}
\frac{\partial}{\partial t}[\varrho(\boldsymbol{x}, t) \boldsymbol{s}(\boldsymbol{x}, t)]+\nabla_{x} \cdot\left[\varrho(\boldsymbol{x}, t) \boldsymbol{v}(\boldsymbol{x}, t) \boldsymbol{s}(\boldsymbol{x}, t)-\mathbf{W}^{\top}(\boldsymbol{x}, t)\right] \\
=\varepsilon: \mathbf{T}(\boldsymbol{x}, t)+\varrho(\boldsymbol{x}, t) \boldsymbol{g}(\boldsymbol{x}, t) .
\end{gathered}
$$




\subsection{Macroscopic total energy}

$$
\begin{aligned}
& e(\boldsymbol{x}, t):=\frac{1}{2} \boldsymbol{v}^{2}(\boldsymbol{x}, t)+\frac{1}{2} \boldsymbol{s}(\boldsymbol{x}, t) \cdot \Theta^{-1} \cdot \boldsymbol{s}(\boldsymbol{x}, t)+\varepsilon(\boldsymbol{x}, t) \\
& \frac{\partial}{\partial t}[\varrho(\boldsymbol{x}, t) e(\boldsymbol{x}, t)]+\nabla_{x} \cdot\left[\boldsymbol{v}(\boldsymbol{x}, t) \varrho(\boldsymbol{x}, t) e(\boldsymbol{x}, t)-\mathbf{T}^{\top}(\boldsymbol{x}, t) \cdot \boldsymbol{v}(\boldsymbol{x}, t)\right. \\
&\left.-\mathbf{W}^{\top}(\boldsymbol{x}, t) \cdot \Theta^{-1} \cdot \boldsymbol{s}(\boldsymbol{x}, t)+\boldsymbol{q}(\boldsymbol{x}, t)\right] \\
&=\varrho(\boldsymbol{x}, t) \boldsymbol{k}(\boldsymbol{x}, t) \cdot \boldsymbol{v}(\boldsymbol{x}, t) \\
& \quad+\varrho(\boldsymbol{x}, t) \boldsymbol{g}(\boldsymbol{x}, t) \cdot \Theta^{-1} \cdot \boldsymbol{s}(\boldsymbol{x}, t)+\varrho(\boldsymbol{x}, t) r(\boldsymbol{x}, t) .
\end{aligned}
$$

Here $\varepsilon(\boldsymbol{x}, t)$ is the macroscopic specific internal energy and $r(\boldsymbol{x}, t)$ the macroscopic absorption supply. The macroscopic heat flux density is $\boldsymbol{q}(\boldsymbol{x}, t)$.

\subsection{Macroscopic internal energy}

$$
\begin{aligned}
\frac{\partial}{\partial t}[\varrho(\boldsymbol{x}, t) \varepsilon(\boldsymbol{x}, t)]+\nabla_{x} \cdot[\varrho(\boldsymbol{x}, t) \boldsymbol{v}(\boldsymbol{x}, t) \varepsilon(\boldsymbol{x}, t)+\boldsymbol{q}(\boldsymbol{x}, t)] \\
=\varrho(\boldsymbol{x}, t) r(\boldsymbol{x}, t)-\boldsymbol{s}(\boldsymbol{x}, t) \cdot \Theta^{-1} \cdot \boldsymbol{\varepsilon}: \mathbf{T}(\boldsymbol{x}, t)+\mathbf{T}^{\top}(\boldsymbol{x}, t):\left[\nabla_{x} \boldsymbol{v}(\boldsymbol{x}, t)\right] \\
\quad+\mathbf{W}(\boldsymbol{x}, t)^{\top}: \nabla_{x}\left(\Theta^{-1} \cdot \boldsymbol{s}(\boldsymbol{x}, t)\right) .
\end{aligned}
$$

The macroscopic balance equations of micropolar media do not represent a five-field theory, because the spin density is included as an additional field in conventional treatment. Consequently, micropolar media are described by a macroscopic eightfield theory. If the spin density is set to zero, we rediscover five-field hydrodynamics.

\subsection{Comparing mesoscopic and macroscopic balances}

The more formal setting by the axiom (20) can be correctly derived, if the mesoscopic balances are integrated over the mesoscopic variables [12],

$$
\int \ldots d \mathscr{M} \rightarrow \text { balances of micropolar media. }
$$

In the case of liquid crystals, this integration is over the different orientations of the uniaxial molecules. Because the microscopic directors span a unit sphere, the integration is over this unit sphere.

A comparison between the mesoscopic balance equations (7) and the macroscopic balances (20) needs first of all the definitions of macroscopic quantities by their mesoscopic back-ground, as we have defined already the macroscopic mass density by (5).

$\square$ Definition: The following mesoscopic fields are additive: 


$$
\begin{aligned}
& \varrho(\boldsymbol{x}, t):=\int \varrho(\cdot) d \mathscr{M}, \quad \boldsymbol{v}(\boldsymbol{x}, t):=\int f(\cdot) \boldsymbol{v}(\cdot) d \mathscr{M} . \\
& \boldsymbol{s}(\boldsymbol{x}, t):=\int f(\cdot) \boldsymbol{s}(\cdot) d \mathscr{M}, \quad e(\boldsymbol{x}, t):=\int f(\cdot) e(\cdot) d \mathscr{M} .
\end{aligned}
$$

Using these definitions and the transport theorem

$$
\frac{\partial}{\partial t} \int \Phi(\cdot) d \mathscr{M}=\int\left[\partial_{l} \Phi(\cdot)+\nabla_{m} \cdot \boldsymbol{u}(\cdot) \Phi(\cdot)\right] d \mathscr{M}
$$

a direct comparison between the mesoscopic balances integrated over $\mathscr{M}$ and the macroscopic ones result in:

\section{$\square$ Proposition:}

$$
\begin{aligned}
& \mathbf{T}(\boldsymbol{x}, t)=\int[\mathbf{T}(\cdot)-\boldsymbol{v}(\cdot) \varrho(\cdot) \boldsymbol{v}(\cdot)] d \mathscr{M}+v(\boldsymbol{x}, t) \varrho(\boldsymbol{x}, t) \boldsymbol{v}(\boldsymbol{x}, t), \\
& \varrho(\boldsymbol{x}, t) \boldsymbol{k}(\boldsymbol{x}, t)=\int\left[\varrho(\cdot) \boldsymbol{k}(\cdot)+\nabla_{m} \mathscr{T}^{\top}(\cdot)\right] d \mathscr{M}, \\
& \mathbf{W}(\boldsymbol{x}, t)=\int[\mathbf{W}(\cdot)-\boldsymbol{s}(\cdot) \varrho(\cdot) \boldsymbol{v}(\cdot)] d \mathscr{M}+\boldsymbol{s}(\boldsymbol{x}, t) \varrho(\boldsymbol{x}, t) \boldsymbol{v}(\boldsymbol{x}, t), \\
& \varrho(\boldsymbol{x}, t) \boldsymbol{g}(\boldsymbol{x}, t)=\int\left[\varrho(\cdot) \boldsymbol{g}(\cdot)+\boldsymbol{\varepsilon}: \mathbf{T}(\cdot)-\nabla_{m} \cdot \mathscr{W}^{\top}(\cdot)\right] d \mathscr{M}-\boldsymbol{\varepsilon}: \mathbf{T}(\boldsymbol{x}, t), \\
& \boldsymbol{q}(\boldsymbol{x}, t)=\int\left[\boldsymbol{q}(\cdot)+\boldsymbol{v}(\cdot) \varrho(\cdot) e(\cdot)-\mathbf{T}^{\boldsymbol{\top}}(\cdot) \cdot \boldsymbol{v}(\cdot)-\mathbf{W}^{\top}(\cdot) \cdot \Theta^{-1} \cdot \boldsymbol{s}(\cdot)\right] d \mathscr{M} \\
& -\boldsymbol{v}(\boldsymbol{x}, t) \varrho(\boldsymbol{x}, t) e(\boldsymbol{x}, t)+\mathbf{T}^{\top}(\boldsymbol{x}, t) \cdot \boldsymbol{v}(\boldsymbol{x}, t) \\
& +\mathbf{W}^{\top}(\boldsymbol{x}, t) \cdot \Theta^{-1} \cdot \boldsymbol{s}(\boldsymbol{x}, t), \\
& \varrho(\boldsymbol{x}, t) r(\boldsymbol{x}, t)=\int\left\{\varrho(\cdot) r(\cdot)+\varrho(\cdot) \boldsymbol{k}(\cdot) \cdot \boldsymbol{v}(\cdot)+\varrho(\cdot) \boldsymbol{g}(\cdot) \cdot \Theta^{-1} \cdot \boldsymbol{s}(\cdot)\right. \\
& \left.+\nabla_{m} \cdot\left[\mathscr{T}^{\top}(\cdot) \cdot \boldsymbol{v}(\cdot)-\mathscr{W}^{\top}(\cdot) \cdot \Theta^{-1} \cdot \boldsymbol{s}(\cdot)+\boldsymbol{Q}(\cdot)\right]\right\} d \mathscr{M} \\
& -\varrho(\boldsymbol{x}, t) \boldsymbol{k}(\boldsymbol{x}, t) \cdot \boldsymbol{v}(\boldsymbol{x}, t)-\varrho(\boldsymbol{x}, t) \boldsymbol{g}(\boldsymbol{x}, t) \cdot \Theta^{-1} \cdot \boldsymbol{s}(\boldsymbol{x}, t), \\
& \varrho(\boldsymbol{x}, t) \varepsilon(\boldsymbol{x}, t)=\int\left[\varrho(\cdot) \varepsilon(\cdot)+\frac{1}{2} \varrho(\cdot) \boldsymbol{v}^{2}(\cdot)+\frac{1}{2} \varrho(\cdot) \boldsymbol{s}(\cdot) \cdot \Theta^{-1} \cdot \boldsymbol{s}(\cdot)\right] d \mathscr{M} \\
& -\frac{1}{2} \varrho(\boldsymbol{x}, t) \boldsymbol{v}^{2}(\boldsymbol{x}, t)-\frac{1}{2} \varrho(\boldsymbol{x}, t) \boldsymbol{s}(\boldsymbol{x}, t) \cdot \Theta^{-1} \cdot \boldsymbol{s}(\boldsymbol{x}, t) .
\end{aligned}
$$


More details about the connection between mesoscopic and macroscopic fields can be found in [11].

\section{Mesoscopic distribution function}

\subsection{Balance of the MDF}

The mesoscopic distribution function (MDF) satisfies a balance equation because of the definition (4) and of the mesoscopic mass balance (9). A straight forward calculation results in [12].

\section{$\square$ Proposition:}

$$
\begin{aligned}
\frac{\partial}{\partial t} f(\cdot) & +\nabla_{x} \cdot[\boldsymbol{v}(\cdot) f(\cdot)]+\nabla_{m} \cdot[\boldsymbol{u}(\cdot) f(\cdot)] \\
& +f(\cdot)\left[\frac{\partial}{\partial t}+\boldsymbol{v}(\cdot) \cdot \nabla_{x}\right] \ln \varrho(\boldsymbol{x}, t)=0 .
\end{aligned}
$$

This balance equation of the MDF contains the macroscopic mass density. Inserting it we can see that (40) is highly non-linear. The macroscopic influence on mesoscopic quantities is often denoted as a "mean field" theory.

In the case of liquid crystals, the MDF is the orientation distribution function (ODF). The mesoscopic variable $\boldsymbol{m}$ becomes the microscopic director $\boldsymbol{n}$, and the manifold $\mathscr{M}$ is the unit sphere $\mathbb{S}^{2}$. According to the definition (2), the ODF describes the local alignment of the molecules. If there is no alignment, which means the molecules are locally isotropically distributed, the ODF becomes independent of $\boldsymbol{n}$. Then the normalization condition (2) results in $f(\boldsymbol{x}, t)=1 /(4 \pi)$ which is called isotropic ODF. If the ODF has an axis of rotational symmetry, a macroscopic director $\boldsymbol{d}$ can be introduced (see Section 6.2). This case is called the uniaxial ODF. All other possible cases which are neither isotropic nor uniaxial are called biaxial. This name has nothing to do with the fact that the molecules themselves may have a uniaxial or biaxial shape (see Section 9). Consequently the expression biaxial has two meanings depending on its reference to the ODF or to the molecules of which the liquid crystal consists.

\subsection{Orientation dynamics}

In the case of liquid crystals, the spin density $s(\cdot)$ is due to particle rotations and therefore determined by the orientation change velocity $\boldsymbol{u}(\cdot)$. In this case the balance of spin can be interpreted as the differential equation for the orientation change velocity. We here will solve the stationary balance of spin (13) and insert the resulting orientation change velocity into the orientation dynamics (40) in order to have a closed differential equation for the distribution function [32]. In this example it is assumed that molecules of different orientation have the same velocity $(\boldsymbol{v}(\cdot)=\boldsymbol{v}(\boldsymbol{x}, t))$ and that the liquid crystal is uniform, i.e., it is homogeneous, except for the macro- 
scopic flow field which has a constant velocity gradient. External fields are excluded, although electric fields and also gradients of the orientational order can be included, too $[33,34]$. The essential simplifying assumption is that the spin is stationary $\partial_{t} s(\cdot)=0$. This presupposition reduces the balance of spin to an algebraic equation for the orientation change velocity $\boldsymbol{u}(\cdot)$ [35],

$$
\rho(\cdot) \boldsymbol{u}(\cdot) \cdot \nabla_{m} \boldsymbol{s}(\cdot)=\varepsilon: \mathbf{T}(\cdot)
$$

The mesoscopic stress tensor $\mathbf{T}(\cdot)$ is a constitutive quantity which therefore is defined on the state space (43) which depends on the considered material. From (41) we see that $\varepsilon: \mathbf{T}$ vanishes, if $\nabla_{n} \boldsymbol{s}(\cdot)=0$. Therefore we can write a constitutive equation of the form

$$
\boldsymbol{\varepsilon}: \boldsymbol{T}(\cdot)=\boldsymbol{G}(\cdot) \cdot \nabla_{n} \boldsymbol{s}(\cdot)
$$

The mesoscopic quantity $\boldsymbol{G}(\cdot)$ is a constitutive one which is defined on the state space, the domain of the constitutive functions. There are different possibilities for introducing state spaces in mesoscopic theory with respect to including mesoscopic and macroscopic state variables (see Section 8). Here the state space has to include mesoscopic variables, because we are in a mesoscopic description. But also macroscopic state variables such as temperature, velocity gradient and the second order alignment tensor are included:

$$
Z=\left(\boldsymbol{n}, \rho(\cdot), \nabla_{n} \ln \varrho(\cdot), \varrho(\boldsymbol{x}, t), T(\boldsymbol{x}, t), \nabla \boldsymbol{v}(\boldsymbol{x}, t), \boldsymbol{a}(\boldsymbol{x}, t)\right) .
$$

The influence of the second order alignment tensor can be interpreted as the orienting effect of the orientational order of the surrounding particles. The orientation gradient of the mesoscopic mass density describes orientation diffusion. Finally, a representation theorem for $\boldsymbol{G}(\cdot)$, being linear in all derivatives, gives

$$
\boldsymbol{u}=\boldsymbol{\omega} \times \boldsymbol{n}+\beta_{3} \nabla_{n} \ln \varrho(\cdot)+(1-\boldsymbol{n n}) \cdot\left(\beta_{1} \overparen{\nabla_{x} \boldsymbol{v}}+\beta_{5} \boldsymbol{a}\right) \cdot \boldsymbol{n} .
$$

Here $\beta_{3}, \beta_{4}$ and $\beta_{5}$ are material coefficients, depending on macroscopic mass density and temperature, and $\omega$ is the angular velocity between material and observer. Here the bracket $\overline{\boldsymbol{a} \boldsymbol{b}}$ is the symmetric traceless part of the tensor $\boldsymbol{a} \boldsymbol{b}$ [8]. Inserting (44) into the orientation balance (40) leads to the following differential equation for the ODF:

$$
\begin{array}{r}
\frac{d}{d t} f(\cdot)+\beta_{3} \nabla_{n} \cdot \nabla_{n} f-3 f \boldsymbol{n} \cdot\left\{\beta_{4} \overparen{\nabla_{x} \boldsymbol{v}}+\beta_{5} \boldsymbol{a}\right\} \cdot \boldsymbol{n} \\
+\left[\boldsymbol{\omega} \times \boldsymbol{n}+\boldsymbol{n} \cdot\left\{\beta_{4} \overparen{\nabla_{x} \boldsymbol{v}}+\beta_{5} \boldsymbol{a}\right\}\right] \cdot \nabla_{n} f=0 .
\end{array}
$$

This differential equation is of the Fokker-Planck type. Orientation diffusion, influence of the mean field and the orienting effect of the flow field are included. This is the same form of an equation derived by S. Hess [36] by a very different approach. 
Here, Eq. (45) is systematically derived in the framework of mesoscopic theory by starting out with the mesoscopic balance equations for mass and spin and a constitutive ansatz on a chosen state space.

\section{Macroscopic fields of order parameters}

The mesoscopic concept introduces the following fields which are beyond the macroscopic description: the mesoscopic variables $\boldsymbol{m}$ included in the mesoscopic space, the MDF $f(\cdot)$, and the mesoscopic change velocity $\boldsymbol{u}(\cdot)$. The form of the mesoscopic balances is as in the macroscopic description, but now defined on mesoscopic space. The mesoscopic background with its greater information in comparison with the macroscopic theory has of course influence on the macroscopic description. We obtain from the mesoscopic background a family of macroscopic fields, the so-called order parameters which cannot be obtained systematically without the mesoscopic background. They are defined as moments of the MDF

$$
\begin{aligned}
\boldsymbol{A}(\boldsymbol{x}, t) & :=\int f(\cdot) \boldsymbol{m} d \mathscr{M}, \\
\boldsymbol{a}(\boldsymbol{x}, t) & :=\int f(\cdot) \overline{\boldsymbol{m} \boldsymbol{m}} d \mathscr{M}, \\
\underline{\boldsymbol{a}}_{4}(\boldsymbol{x}, t) & :=\int f(\cdot) \widetilde{\boldsymbol{m m m m}} d \mathscr{M}, \\
\underline{\boldsymbol{a}}_{N}(\boldsymbol{x}, l) & :=\int f(\cdot) \widetilde{\boldsymbol{m} \ldots \mathrm{N} \text { times } \ldots \boldsymbol{m}} d \mathscr{M} .
\end{aligned}
$$

\subsection{Alignment tensors}

In the case of liquid crystals of uniaxial molecules, the mesoscopic variable $\boldsymbol{m}$ is the microscopic director $\boldsymbol{n}(\boldsymbol{n} \cdot \boldsymbol{n}=1)$, and the MDF becomes the orientation distribution function $(\mathrm{ODF})$, which can be expanded into a series of multipoles $[12,8]$

$$
f(\boldsymbol{n}, \boldsymbol{x}, t)=\frac{1}{4 \pi}\left(1 + \sum _ { l = 1 } ^ { \infty } ( 2 l - 1 ) ! ! a _ { \mu _ { 1 } \ldots \mu _ { l } } ( \boldsymbol { x } , t ) \longdiv { n _ { \mu _ { 1 } } \cdots n _ { \mu _ { l } } }\right)
$$

Here is

$$
l ! !=l(l-2)(l-4) \cdots
$$

Greek indices $\mu_{1}, \ldots, \mu_{l}$ denote the components of the microscopic director with respect to a cartesian basis and $\longmapsto$ marks the (totally) symmetric irreducible part of a tensor, that means, for a given tensor $b_{\mu_{1} \ldots \mu_{l}}$ the part $b_{\mu_{1} \ldots \mu_{l}}$ is symmetric with respect to index permutations and will vanish for arbitrary contractions $\left(b_{\mu \mu \mu_{3} \ldots \mu_{l}}=0\right)$. 
As done in (50), we use Einsteins summation convention, but we suppress the distinction between co- and contravariant indices which can be identified in a cartesian basis. The tensorial coefficients $a_{\mu_{1} \ldots \mu_{l}}(\boldsymbol{x}, t)$ in (50) which are analogous to (49) are called the alignment tensors of order $l$ : they are given by

$$
a_{\mu_{1} \ldots \mu_{l}}(\boldsymbol{x}, t): = \frac { 2 l + 1 } { l ! } \oint _ { S ^ { 2 } } f ( \cdot ) \longdiv { n _ { \mu _ { 1 } } \cdots n _ { \mu _ { l } } } d ^ { 2 } n
$$

For uniaxial molecules the ODF shows the so-called head-tail symmetry or nematic symmetry

$$
f(\boldsymbol{n}, \boldsymbol{x}, t)=f(-\boldsymbol{n}, \boldsymbol{x}, t)
$$

which results from the fact that head and tail of the considered molecules are indistinguishable. Because of the head-tail symmetry (53), the moments of odd order of the ODF vanish in (49) and in (52). Thus in the case of liquid crystals, only the alignment tensors of even order can be different from zero. Interesting is, that there is no alignment tensor of the first order which is different from zero, which means we have to define in mesoscopic theory what a macroscopic director used in director theories of liquid crystals may be (see Section 6.2).

Using definition (52) the eigenvalues of the alignment tensor of second order can be estimated easily [27].

$\square$ Proposition: Because of (52) and (53) we obtain for the eigenvalues $\lambda$ of the alignment tensor of second order $\boldsymbol{a}$ the following estimations

$$
\lambda_{\text {max }} \leq \frac{2}{3}, \quad \lambda_{\text {min }} \geq-\frac{1}{3}
$$

\subsection{The macroscopic director}

The easiest case to define a macroscopic director is if the ODF has an axis of rotational symmetry, and a unit vector $\boldsymbol{e}$ parallel to this symmetry axis. This case is often called uniaxial. This name has nothing to do with the property of the molecules to be uniaxial or biaxial. "Uniaxial" here refers to the rotational symmetry of the ODF. If we denote the angle between $\boldsymbol{e}$ and the microscopic director $\boldsymbol{n}$ by $\Theta$, the mesoscopic part of the ODF depends only on $\cos \Theta$ because of the rotational symmetry

$$
f(\boldsymbol{n}, \boldsymbol{x}, t)=g(\cos \Theta, \boldsymbol{x}, t) .
$$

According to (52) the traceless alignment tensor of the second order is

$$
a _ { \mu _ { 1 } \mu _ { 2 } } ( \boldsymbol { x } , t ) = \frac { 5 } { 2 } \oint _ { S ^ { 2 } } \boldsymbol { g } ( \operatorname { c o s } \Theta ) \longdiv { n _ { \mu _ { 1 } } n _ { \mu _ { 2 } } } d ^ { 2 } n
$$

By a straight forward calculation we can prove the following [37]

J. Non-Equilib. Thermodyn. · 2004 · Vol. 29 - No. 1 
$\square$ Proposition: If $\boldsymbol{e}$ is a unit vector parallel to the rotational symmetry axis of the $\mathrm{ODF}$, the alignment tensor of second order is

$$
\begin{aligned}
& \boldsymbol { a } ( \boldsymbol { x } , t ) = \frac { 5 } { 2 } \pi \langle 3 \operatorname { c o s } ^ { 2 } \Theta - 1 \rangle ( \boldsymbol { x } , t ) \longdiv { \boldsymbol { e } ( \boldsymbol { x } , t ) \boldsymbol { e } ( \boldsymbol { x } , t ) } \\
& \left\langle 3 \cos ^{2} \Theta-1\right\rangle(\boldsymbol{x}, t):=\int_{0}^{\pi} d \Theta \sin \Theta g(\cos \Theta, \boldsymbol{x}, t)\left(3 \cos ^{2} \Theta-1\right) .
\end{aligned}
$$

This proposition shows, that in the case of a uniaxial ODF, the shape of the alignment tensor of second order is

$$
\boldsymbol { a } ( \boldsymbol { x } , t ) = S ( \boldsymbol { x } , t ) \longdiv { \boldsymbol { e } ( \boldsymbol { x } , t ) \boldsymbol { e } ( \boldsymbol { x } , t ) }, \quad \boldsymbol{e} \cdot \boldsymbol{e}=1
$$

Hence its eigenvalues and eigenvectors are (see (54))

$$
\begin{array}{ll}
\boldsymbol{a} \cdot \boldsymbol{e}=\frac{2}{3} S \boldsymbol{e}, & \boldsymbol{a} \cdot \boldsymbol{e}_{j}^{\perp}=-\frac{1}{3} S \boldsymbol{e}_{j}^{\perp}, \quad j=1,2, \\
\boldsymbol{e} \cdot \boldsymbol{e}=1, & \boldsymbol{e}_{j}^{\perp} \cdot \boldsymbol{e}_{k}^{\perp}=\delta_{j k}, \quad \boldsymbol{e} \cdot \boldsymbol{e}_{j}^{\perp}=0 .
\end{array}
$$

The unit vector $\boldsymbol{e}$ parallel to the rotational symmetry axis of the ODF which is eigenvector of the corresponding alignment tensor to the greatest eigenvalue $(2 / 3) S$ defines the macroscopic director. This definition makes use of the form (59) of the alignment tensor. Thus in mesoscopic theory the macroscopic director is a derived and not a basic quantity.

Besides the macroscopic director $\boldsymbol{e}$, the scalar order parameter $S$ is also essential for describing liquid crystals [13]. Because of its normalization, the macroscopic director cannot describe changes in the degree of orientational order as they appear during the phase transition nematic-isotropic. These changes in the degree of orientational order are described by the scalar order parameter. Consequently, pure director theory of liquid crystals cannot include this aspect (see Section 7).

Now the question arises, whether in general, that is in the non-uniaxial case, a macroscopic director exists. According to (52) the alignment tensor of second order is traceless, that means, for its three eigenvalues we have

$$
\lambda_{\text {max }} \geq \lambda^{\prime} \geq \lambda_{\text {min }}, \quad \lambda_{\text {max }}+\lambda^{\prime}+\lambda_{\text {min }}=0, \quad \lambda_{\text {max }} \geq 0, \quad \lambda_{\text {min }} \leq 0 .
$$

If we take, as in the rotational symmetric case (60), the eigenvector belonging to the greatest eigenvalue as a macroscopic director, this definition is not unique in two cases: if $\lambda_{\max }=\lambda^{\prime}$ the two positive eigenvalues coincide, or if $\lambda_{\min }=0$ all eigenvalues are zero, which corresponds to the isotropic case. As we can easily see the only remaining possibility for defining a macroscopic director is

$$
\lambda_{\text {min }}<0: \quad \boldsymbol{d}:=\left(1-\delta_{0 \beta}\right) \boldsymbol{e}_{\max }+\delta_{0 \beta} \boldsymbol{e}_{\min }, \quad \beta:=\lambda_{\max }-\lambda^{\prime} .
$$


Because the eigenvalues of the alignment tensor are macroscopic fields, it is possible that the macroscopic director defined by (63) is discontinuous and jumps from $\boldsymbol{e}_{\max }$ to $\boldsymbol{e}_{\min }$ and is not defined in between. This discontinuity has no physical background because the corresponding alignment tensor is continuous. Consequently, it seems to be more satisfactory to use an alignment tensor theory, or even the mesoscopic theory for describing liquid crystals.

\subsection{Balances of order parameters}

According to their definition (49) the order parameters and also the alignment tensors (52) are macroscopic fields. Balance equations for these additional macroscopic fields can be derived as follows: if the k-th cartesian component of $\boldsymbol{m}$ is denoted by $m_{k}$, and if we want to find the equation of motion of the order parameter $\boldsymbol{a}_{\ell}$ of order $\ell$ we multiply (40) by $m_{\mu_{1}} \cdots m_{\mu_{\ell}}$ and integrate over the mesoscopic manifold $\mathscr{M}$. If, additionally, the mesoscopic manifold $\mathscr{M}$ is time independent, and if we introduce the difference between mesoscopic and macroscopic material velocity by

$$
\boldsymbol{v}(\cdot)=\boldsymbol{v}(\boldsymbol{x}, t)+\delta \boldsymbol{v}(\cdot),
$$

we can prove $[20]$ the

\section{$\square$ Proposition:}

$$
\begin{aligned}
& \frac{\partial}{\partial t} \underline{\boldsymbol{a}_{\ell}}+\nabla_{x} \cdot\left[\boldsymbol{v}(\boldsymbol{x}, t) \underline{\boldsymbol{a}_{\ell}}+\int\left[\delta \boldsymbol{v}(\cdot) f(\cdot) \widetilde{m_{\mu_{1}} \cdots m_{\mu_{\ell}}}\right] d \mathscr{M}\right] \\
& +\int \overparen{m_{\mu_{1}} \cdots m_{\mu_{\ell}}} \nabla_{m} \cdot[\boldsymbol{u}(\cdot) f(\cdot)] d \mathscr{M} \\
& +\underline{\boldsymbol{a}_{\ell}}\left[\frac{\partial}{\partial t} \ln \rho(\boldsymbol{x}, t)+\boldsymbol{v}(\boldsymbol{x}, t) \cdot \nabla_{x} \ln \rho(\boldsymbol{x}, t)\right]=0 .
\end{aligned}
$$

In the case of a general mesoscopic manifold $\mathscr{M},(65)$ cannot be simplified further, but we will derive a simplified equation for the example of biaxial liquid crystals in Section 9.

\subsection{Landau-type equation of second order alignment tensor}

According to (47) the second moment of the ODF is the second order alignment tensor. Therefore, multiplying equation (45) with $\overrightarrow{\boldsymbol{n n}}$ and integrating over all orientations results in a differential equation for $\boldsymbol{a}$.

$\square$ Proposition: [35]: Introducing the abbreviation

$$
\boldsymbol{A}:=\beta_{4} \widetilde{\nabla_{x} \boldsymbol{v}}+\beta_{5} \boldsymbol{a}
$$

we obtain from (45)

J. Non-Equilib. Thermodyn. · 2004 · Vol. $29 \cdot$ No. 1 


$$
\frac{d}{d l} \boldsymbol{a}-2 \widetilde{\omega} \times \boldsymbol{a}=6 \beta_{3} \boldsymbol{a}+\frac{2}{5} \boldsymbol{A}+\frac{6}{7} \overparen{\boldsymbol{A} \cdot \boldsymbol{a}}-2 \overrightarrow{\boldsymbol{A}: \underline{\boldsymbol{a}_{4}}} .
$$

In this equation the fourth moment $\boldsymbol{a}_{4}$ of the distribution function appears, for which we need a closure relation. Here we take advantage of the often used simple ansatz for the fourth non-traceless moment

$$
\int_{S^{2}} f(\cdot) \boldsymbol{n n n n n} d^{2} n=\int_{S^{2}} f(\cdot) \boldsymbol{n n} d^{2} n \int_{S^{2}} f(\cdot) \boldsymbol{n} \boldsymbol{n} d^{2} n .
$$

$\square$ Proposition [6]: Inserting (68) into (67) we obtain for the vanishing velocity gradient the equation of motion in the following form

$$
\frac{d}{d t} \boldsymbol{a}=\frac{d}{d \boldsymbol{a}} \Psi
$$

Here the right hand side of this equation is the derivative of the free energy density $\Psi$.

This is exactly the form of the equation of motion assumed in the dynamical Landau-theory $[38,39]$. The corresponding Landau free energy density is

$$
\Psi=\left(3 \beta_{3}+\frac{1}{5} \beta_{5}\right) \boldsymbol{a}: \boldsymbol{a}+\frac{2}{7} \beta_{5} \operatorname{Tr}(\boldsymbol{a} \cdot \boldsymbol{a} \cdot \boldsymbol{a})-\frac{1}{2} \beta_{5}(\boldsymbol{a}: \boldsymbol{a})^{2} .
$$

For a non-vanishing velocity gradient it is known that such a derivation of the time rate of the alignment tensor from a potential is only possible in very special flow geometries, but not in general [40].

On the other hand, the same type of equation (66) has also been derived from an exploitation of the dissipation inequality with methods of Irreversible Thermodynamics $[6,41]$.

\section{Ericksen-Leslie theory}

\subsection{Mesoscopic back-ground}

The Ericksen-Leslie theory of liquid crystals of uniaxial molecules uses a macroscopic director field $\boldsymbol{d}(\boldsymbol{x}, t)$ for describing the local alignment of the molecules without any mesoscopic background as discussed in Section (6.2). Here we now investigate the conditions necessary to go from the mesoscopic theory of liquid crystals to Ericksen-Leslie theory.

The mesoscopic spin of a needleshaped molecule is

$$
\boldsymbol{s}(\cdot)=\boldsymbol{I n} \times \boldsymbol{u}(\cdot)=: \boldsymbol{I} \boldsymbol{\omega}(\cdot) .
$$


Here $\boldsymbol{I}$ is the moment of inertia belonging to an axis of rotation perpendicular to the microscopic director $\boldsymbol{n}$ of a single molecule. We now can define the non-objective "energy of rotation" by

$$
u_{r}(\cdot):=\frac{1}{2} I[\boldsymbol{n} \times \boldsymbol{u}(\cdot)]^{2}=\frac{1}{2} I \omega^{2}(\cdot) .
$$

Multiplication with the ODF and integration over the mesoscopic manifold, here the unit sphere spanned locally by all possible microscopic directors, yield the macroscopic field of "rotation energy"

$$
u_{r}(\boldsymbol{x}, t):=\frac{1}{2} I \oint_{S^{2}} f(\cdot) \omega^{2}(\cdot) d^{2} n
$$

From (71) and (72) we get a relation between spin and rotation energy

$$
s^{2}(\cdot)=2 \operatorname{Iu}(\cdot)
$$

which is of course not valid for the macroscopic fields (31) and (73) for which the inequality

$$
\boldsymbol{s}^{2}(\boldsymbol{x}, t) \leq 2 u_{r}(\boldsymbol{x}, t)
$$

holds. This inequality is easy to prove. From (31) we obtain

$$
\boldsymbol{s}^{2}(\boldsymbol{x}, t)=I^{2}\left\{\oint[\sqrt{f(\cdot)}][\sqrt{f(\cdot)} \boldsymbol{\omega}(\cdot)] d^{2} n\right\}^{2},
$$

and by use of Schwarz inequality [42] (76) results in

$$
\boldsymbol{s}^{2}(\boldsymbol{x}, t) \leq I^{2} \oint f(\cdot) d^{2} n \oint f(\cdot) \omega^{2}(\cdot) d^{2} n
$$

from which (75) follows immediately by (73) and by the normalization of the ODF. As we can prove, the inequality (75) is observer-independent [3]. Consequently, it must be valid for all macroscopic liquid crystal theories, including that of EricksenLeslie.

We now will investigate, if and on what conditions (75) is satisfied in Ericksen-Leslie theory.

\subsection{Macroscopic spin and characterization}

Because the Ericksen-Leslie theory has no mesoscopic background, there is no motivation to introduce a microscopic director $\boldsymbol{n}$ and its ODF. Instead of that a mesoscopically undefined normalized macroscopic director field $\boldsymbol{d}(\boldsymbol{x}, l)$ is used $[1,2,43]$ defining spin and rotation energy by 


$$
\begin{aligned}
& \boldsymbol{s}^{E L}(\boldsymbol{x}, t):=\operatorname{Id}(\boldsymbol{x}, t) \times \partial_{t} \boldsymbol{d}(\boldsymbol{x}, t) \\
& u_{r}^{E L}(\boldsymbol{x}, t):=\frac{1}{2} I\left[\boldsymbol{d}(\boldsymbol{x}, t) \times \partial_{t} \boldsymbol{d}(\boldsymbol{x}, t)\right]^{2} \\
& \boldsymbol{d}^{2}(\boldsymbol{x}, t)=1 .
\end{aligned}
$$

Consequently,

$$
\left[\boldsymbol{s}^{E L}(\boldsymbol{x}, t)\right]^{2}=2 \operatorname{Iu}_{r}^{E L}(\boldsymbol{x}, t)
$$

follows for the macroscopic fields, as (74) follows for single molecules mesoscopically, which is different from the inequality (75). Therefore we give the

$\square$ Definition: Ericksen-Leslie theory of liquid crystals is characterized by the field of a macroscopic director $\boldsymbol{d}(\boldsymbol{x}, t)$ and by the definitions (78) to (80) for macroscopic spin and rotation energy.

Consequently, Ericksen-Leslie theory is the special case which satisfies just the equality in (75). From this fact a mesoscopic characterization for the validity of Ericksen-Leslie theory follows by the

$\square$ Proposition [3]: Ericksen-Leslie theory is only valid, if the molecules are local totally aligned in the directions $\boldsymbol{d}$ and $-\boldsymbol{d}$

$$
f(\cdot) \boldsymbol{u}(\cdot)=\frac{1}{2}[\delta(\boldsymbol{n}-\boldsymbol{d}(\boldsymbol{x}, t))-\delta(\boldsymbol{n}+\boldsymbol{d}(\boldsymbol{x}, t))] \partial_{t} \boldsymbol{d}(\boldsymbol{x}, t)
$$

$\left(\delta(\cdot)\right.$ is the $\delta$-function), or if they form a global planar phase ${ }^{2)}$ characterized by

$$
\begin{aligned}
& \boldsymbol{n} \cdot \boldsymbol{C}(t)=0 \text { for all }(\boldsymbol{n}, \boldsymbol{x}, t), \\
& \boldsymbol{s}(\cdot)=\boldsymbol{s}^{E L}(\boldsymbol{x}, t)=I \boldsymbol{C}(t)
\end{aligned}
$$

This result, that the Ericksen-Leslie theory is only valid if the molecules are totally aligned, can be elucidated very clearly: if we have a uniaxial ODF and choose its rotational symmetry axis as a macroscopic director $\boldsymbol{d}$, a rotation around the macroscopic director does not change its direction $\partial_{t} \boldsymbol{d}=\mathbf{0}$. Consequently, the spin belonging to a uniaxial ODF is always zero in Ericksen-Leslie theory. Because the spin of such a configuration is obviously not zero, the Ericksen-Leslie theory does not fit for the uniaxial class of ODFs.

If now all molecules are totally aligned along $\boldsymbol{d}$ (or $-\boldsymbol{d}$ ), the spin belonging to this $\delta$-shaped ODF is indeed zero (no spin by rotation around the long axis of the molecules). Here the Ericksen-Leslie theory of one-director description holds true. Whenever the deviation of the molecules from the total alignment can be ignored, the Ericksen-Leslie theory is a sufficient description generated as that special case of the mesoscopic theory in which the ODF (82) is valid.

\footnotetext{
${ }^{2)}$ There is a hint [44] that such a planar phase does not appear in nature.
} 


\section{Constitutive properties}

\subsection{General remarks}

The balance equations in the macroscopic as well as in the mesoscopic case are underdetermined, because they are valid for arbitrary materials. Consequently, constitutive equations are needed in order to close the system of balance equations. On the mesoscopic level there are even more constitutive equations than on the macroscopic one, because there are additional fluxes in mesoscopic space. Also for the state space, the domain of the constitutive mappings, there are more different choices possible than in the macroscopic case: the state space can either include only mesoscopic variables, or mesoscopic and macroscopic variables both together.

The mesoscopic constitutive theory has not been systematically well developed up to now. The difficulties which appear become clear, if we consider the state space (43) we used in Section 5.2. This state space is a mixed one, consisting of mesoscopic and macroscopic variables. Obviously, in a mesoscopic theory mesoscopic variables must be included in the state space. But how to introduce also macroscopic variables describing "mean field" effects of the surrounding molecules is not evident at first.

The constitutive mappings defined on the mesoscopic level are not restricted by the second law of thermodynamics, because the mesoscopic entropy production is not necessarily positive definite. An exploitation of the dissipation inequality with respect to restrictions on constitutive functions is possible only on the macroscopic level. However, the macroscopic state space can include macroscopic quantities which are defined by the mesoscopic background [45].

Mesoscopic and macroscopic constitutive theory are not independent of each other. The macroscopic constitutive quantities are averages over mesoscopic ones, but in the case of fluxes, other mesoscopic quantities also contribute, as discussed in Section 4.7. In general, a constitutive ansatz on the mesoscopic level and the averaging procedure results in a macroscopic constitutive equation containing additional information which is not available from purely macroscopic considerations.

In the next section we will discuss as an example the dependence of the macroscopic viscosity coefficients on the mesoscopic order parameters.

\subsection{Example: Anisotropic viscosity}

First of all we split the gradient of velocity as usual into three parts

$$
\begin{aligned}
& \nabla_{x} \boldsymbol{v}=\frac{1}{3}\left(\nabla_{x} \cdot \boldsymbol{v}\right) 1+\overparen{\nabla_{x} \boldsymbol{v}}+\omega, \\
& \overparen{\nabla_{x} \boldsymbol{v}}:=\frac{1}{2}\left[\nabla_{x} \boldsymbol{v}+\nabla_{x} \boldsymbol{v}^{\top}\right]-\frac{1}{3}\left(\nabla_{x} \cdot \boldsymbol{v}\right) 1, \\
& \omega:=\frac{1}{2}\left[\nabla_{x} \boldsymbol{v}-\left(\nabla_{x} \boldsymbol{v}\right)^{\top}\right] .
\end{aligned}
$$


Here $\overparen{\nabla_{x} v}$ is the symmetric traceless part, $\omega$ the antisymmetric part and the $\nabla_{x} \cdot \boldsymbol{v}$ the trace of $\nabla_{x} \boldsymbol{v}$.

The Cauchy stress tensor is split into

$$
\mathbf{T}=-p 1-\mathbf{P}
$$

and the co-rotational time derivative of the macroscopic director is

$$
\boldsymbol{N}=\frac{d}{d t} \boldsymbol{d}-\omega \times \boldsymbol{d}, \quad \omega:=\varepsilon: \omega
$$

Now we have to write down the macroscopic constitutive equation for the dissipative part of the stress tensor by the ansatz [37, 46, 47]

$$
\begin{aligned}
-\mathbf{P}(\boldsymbol{x}, t)= & \alpha_{1} \boldsymbol{d} \boldsymbol{d} \boldsymbol{d} \cdot \widetilde{\nabla_{x} \boldsymbol{v}} \cdot \boldsymbol{d}+\alpha_{2} \boldsymbol{d} \boldsymbol{N}+\alpha_{3} \boldsymbol{N} \boldsymbol{d} \\
& +\alpha_{4} \overline{\nabla_{x} \boldsymbol{v}}+\alpha_{5} \boldsymbol{d} \overline{\nabla_{x} \boldsymbol{v}} \cdot \boldsymbol{d}+\alpha_{6} \boldsymbol{d} \cdot \overline{\nabla_{x} \boldsymbol{v} \boldsymbol{d}} \\
& +\zeta_{1} \boldsymbol{d} \cdot \overline{\nabla_{x} \boldsymbol{v}} \cdot \boldsymbol{d} 1+\zeta_{2}\left(\nabla_{x} \cdot \boldsymbol{v}\right) \boldsymbol{d} \boldsymbol{d}+\zeta_{3}\left(\nabla_{x} \cdot \boldsymbol{v}\right) 1
\end{aligned}
$$

Here the viscosities $\alpha_{k}$ and $\zeta_{j}$ are constant, whereas $\boldsymbol{v}(\boldsymbol{x}, t), \boldsymbol{d}(\boldsymbol{x}, t)$ and $\boldsymbol{N}(\boldsymbol{x}, t)$ are macroscopic fields. Because scalar order parameters do not appear in (90), the macroscopic director is related to the Ericksen-Leslie theory of total alignment (see Section 7.2). Consequently, the viscosities $\alpha_{k}$ and $\zeta_{j}$ are Ericksen-Leslie viscosities belonging to total alignment.

The mesoscopic constitutive equation for the dissipative part of the stress tensor is now generated by analogy. The macroscopic quantities in (90) are replaced by the mesoscopic ones [48], which means we replace

$$
\boldsymbol{d}(\boldsymbol{x}, t) \rightarrow \boldsymbol{n}, \quad \boldsymbol{v}(\boldsymbol{x}, t) \rightarrow \boldsymbol{v}(\cdot) .
$$

For simplicity the orientation diffusion is ignored. Hence, $\boldsymbol{v}(\cdot)$ is not introduced, and $\boldsymbol{v}(\boldsymbol{x}, t)$ and $\boldsymbol{\omega}(\boldsymbol{x}, t)$ remain in the mesoscopic case as in the macroscopic one. The corotational change of the macroscopic director (89) is replaced by that of the microscopic one

$$
\boldsymbol{N}(\cdot)=\boldsymbol{u}(\cdot)-\boldsymbol{\omega}(\boldsymbol{x}, t) \times \boldsymbol{n} .
$$

Here $\boldsymbol{u}(\cdot)$ is the mesoscopic orientation change velocity. Consequently, (90) transforms to

$$
\begin{aligned}
& -\mathbf{P}(\cdot)=f(\cdot)\left\{\alpha_{1} \boldsymbol{n n n} \cdot \widetilde{\nabla_{x} \boldsymbol{v}(\boldsymbol{x}, t)} \cdot \boldsymbol{n}+\alpha_{2} \boldsymbol{n} \boldsymbol{N}(\cdot)+\alpha_{3} \boldsymbol{N}(\cdot) \boldsymbol{n}\right. \\
& +\alpha_{4} \overparen{\nabla_{x} \boldsymbol{v}(\boldsymbol{x}, t)}+\alpha_{5} \boldsymbol{n} \overparen{\nabla_{x} \boldsymbol{v}(\boldsymbol{x}, t)} \cdot \boldsymbol{n}+\alpha_{6} \boldsymbol{n} \cdot \overparen{\nabla_{x} \boldsymbol{v}(\boldsymbol{x}, t)} \boldsymbol{n} \\
& \left.+\zeta_{1} \boldsymbol{n} \cdot \overparen{\nabla_{x} \boldsymbol{v}(\boldsymbol{x}, t)} \cdot \boldsymbol{n} 1+\zeta_{2}\left(\nabla_{x} \cdot \boldsymbol{v}(\boldsymbol{x}, t)\right) \boldsymbol{n n}+\zeta_{3}\left(\nabla_{x} \cdot \boldsymbol{v}(\boldsymbol{x}, t)\right) 1\right\} .
\end{aligned}
$$


Because of $\boldsymbol{v}(\cdot)=\boldsymbol{v}(\boldsymbol{x}, t),(33)$ results in

$$
\mathbf{T}(\boldsymbol{x}, t)=\oint_{S^{2}} \mathbf{T}(\cdot) d^{2} n \rightarrow \mathbf{P}(\boldsymbol{x}, t)=\oint_{S^{2}} \mathbf{P}(\cdot) d^{2} n
$$

The ODF in (93) appears, because in case of total alignment we have to rediscover (90) by use of (94).

Now the question arises, what are the vicosities, if the molecules are not totally aligned, for instance in the uniaxial case. Then the ODF in (93) is given by (55) and the alignment tensor (59) becomes, according to (90),

$$
\boldsymbol{a}(\boldsymbol{x}, t)=S_{2}(\boldsymbol{x}, t) \overparen{\boldsymbol{d}(\boldsymbol{x}, t) \boldsymbol{d}(\boldsymbol{x}, t)}, \quad \boldsymbol{d} \cdot \boldsymbol{d}=1,
$$

and the alignment tensor of fourth order is in the uniaxial case

$$
\underline{\boldsymbol{a}_{4}}(\boldsymbol{x}, t)=S_{4}(\boldsymbol{x}, t) \stackrel{\boldsymbol{d}(\boldsymbol{x}, t) \boldsymbol{d}(\boldsymbol{x}, t) \boldsymbol{d}(\boldsymbol{x}, t) \boldsymbol{d}(\boldsymbol{x}, t)}{.}
$$

We now integrate (93) over all orientations, insert (95) and (96), put $\boldsymbol{u}(\cdot)$ approximately to zero, take the Onsager-Parodi relations into account and obtain an expression for $-\mathbf{P}(\boldsymbol{x}, t)$ according to (90) [49]. From this macroscopic expression for the dissipative pressure tensor we can read off the viscosities in the unixial case (marked by ${ }^{u n i}$ ), if the unmarked viscosities belong to the total alignment [49]

$$
\begin{aligned}
& \alpha_{1}^{u n i}=S_{4} \alpha_{1}, \\
& \alpha_{4}^{u n i}=\alpha_{4}+\frac{2}{15}\left(1-\frac{10}{7} S_{2}+\frac{3}{7} S_{4}\right) \alpha_{1}+\frac{1}{3}\left(1-S_{2}\right)\left(\alpha_{5}+\alpha_{6}\right), \\
& \alpha_{5}^{\text {uni }}=S_{4} \alpha_{5}+\frac{2}{7}\left(S_{2}-S_{4}\right) \alpha_{1}, \\
& \alpha_{6}^{\text {uni }}=S_{2} \alpha_{6}+\frac{2}{7}\left(S_{2}-S_{4}\right) \alpha_{1}, \\
& \zeta_{1}^{u n i}=S_{2} \zeta_{1}+\frac{1}{7}\left(S_{2}-S_{4}\right) \alpha_{1}, \\
& \zeta_{2}^{u n i}=S_{2} \zeta_{2}, \quad \zeta_{3}^{u n i}=\zeta_{3}+\frac{1}{3}\left(1-S_{2}\right) \zeta_{2} .
\end{aligned}
$$

Now the material behaviour is characterized by nine material parameters, the coefficients $\alpha_{k}, k=1,4,5,6$, by the order parameters $S_{2}$ and $S_{4}$, and by the viscosities $\zeta_{j}, j=1,2,3$. In experiments usually only the Maier-Saupe order parameter $S_{2}$ is measured, and the $\zeta_{j}$ can be excluded by special experiments. Therefore, in order to compare with experiments, a closure relation $S_{4}\left(S_{2}\right)$ is needed for $S_{4}$. Such a closure relation for higher moments is discussed for instance in [49]. Then the equations (97) to (102) are a theoretical prediction of the order parameter dependence of the material coefficients $\alpha_{k}^{u n i}$. In this case the coefficients of the totally ordered phase $\alpha_{k}$ are fitting parameters to measurements. 
The number of independent coefficients can still be reduced by the affine transformation model $[49,50]$. It is assumed that the molecules are of rotation symmetric ellipsoidal shape with axis ratio $a / b$. A totally ordered fluid, of parallel ellipsoids for example, can be considered as the result of an affine transformation of a fluid of spherical particles, stretching in one direction, and compressing in the two perpendicular ones. A (simple) fluid of spherical particles has two viscosity coefficients: a bulk and a shear viscosity.

Applying the affine transformation, the ordered fluid with five parameters is obtained, but these five parameters depend only on the axis ratio, the bulk and the shear viscosity of the simple reference fluid. In a simple Couette flow, the bulk viscosity is of no importance, and there remain only two parameters: the shear viscosity of the reference fluid and the axis ratio of the particles. With only these two fit parameters, it was possible to fit experimental curves of order parameter dependence very closely for all viscosity coefficients of different substances [49].

\section{Liquid crystals of biaxial molecules}

As reported in Section 6.1 a prominent example for the mesoscopic variable $\boldsymbol{m}$ is the microscopic director $\boldsymbol{n}$ in liquid crystal theory for uniaxial molecules, because the orientation of such a molecule can be described by one direction represented by this microscopic director. In the case of biaxial molecules we need two directions for describing the orientation of one molecule. A constraint between these two directors is the fixed angle between them, usually chosen to be $\pi / 2$. Therefore the orientation of a biaxial molecule is fixed by three parameters, two for the orientation of the first director, a third one for that of the second director [16]. Because of this constraint, a two-director theory $[51,52]$ is clumsy to handle. Therefore it is advantageous to look for another set of mesoscopic variables for describing the orientation of biaxial liquid crystals. This is the subject of this section.

\subsection{Set of mesoscopic variables}

The orientation of a biaxial molecule is described by a proper orthogonal transformation $\mathbf{Q}$ which is defined on a triad of reference and which results in the actual orientation of the molecule [53]. Because $\mathbf{Q}$ is characterized by three parameters, we may choose them as a set of mesoscopic variables. But this set is difficult to handle, and therefore we will replace it by another one. First of all we remember the

$\square$ Proposition (Euler-D'Alembert): Each proper orthogonal transformation on $\mathbb{R}^{3}$ can be described uniquely by a 3-dimensional unit vector $\boldsymbol{n}^{0} \in \mathbb{R}^{3},\left|\boldsymbol{n}^{0}\right|=1$, and an angle $\varphi \in[0, \pi]$, if we identify $\left(\boldsymbol{n}^{0}, \pi\right) \equiv\left(-\boldsymbol{n}^{0}, \pi\right)$ :

$$
\begin{aligned}
& \mathbf{Q} \cdot \mathbf{Q}^{\top}=\mathbf{Q}^{\top} \cdot \mathbf{Q}=1, \quad \mathbf{Q} \cdot \boldsymbol{n}^{0}=\boldsymbol{n}^{0}, \\
& 0=\boldsymbol{n}^{0} \cdot \boldsymbol{z}^{0}=\boldsymbol{n}^{0} \cdot \mathbf{Q}^{\top} \cdot \mathbf{Q} \cdot \boldsymbol{z}^{0}=: \boldsymbol{n}^{0} \cdot \boldsymbol{z}^{0 *}, \\
& \boldsymbol{z}^{0} \cdot \boldsymbol{z}^{0 *}=\cos \varphi .
\end{aligned}
$$


According to this proposition all rotations around an arbitrary, but fixed axis of the direction $\left\{\boldsymbol{n}^{0},-\boldsymbol{n}^{0}\right\}$ are represented by the sets $\left(\boldsymbol{n}^{0}, \varphi\right)$ and $\left(-\boldsymbol{n}^{0}, \varphi\right)$.

The three free parameters of an orthogonal transformation which are included in $\boldsymbol{n}^{0}$ and $\varphi$ can be represented by quaternions [54]. A quaternion $x \in \mathbb{H}^{3)}$ is defined as follows

$$
\begin{aligned}
& x=x_{0}+x_{1} i+x_{2} j+x_{3} k=: x_{0}+\tilde{x} \in \mathbb{H}, \\
& i^{2}=j^{2}=k^{2}=-1, \quad i j=k(c y c l .), \quad j i=-k(c y c l .) .
\end{aligned}
$$

The multiplication is as usual, but non-commutative. The part $x_{0} \in \mathrm{Re} \mathbb{H}$ is called the real and $\tilde{x} \in \operatorname{Im} \mathbb{H}$ is the imaginary part of the quaternion $x$.

We now introduce two (basis-dependent) mappings

$$
\begin{aligned}
& \boldsymbol{V}: \operatorname{Im} \mathbb{H} \rightarrow \mathbb{R}^{3}, \quad \tilde{a} \mapsto \boldsymbol{a}=\boldsymbol{V}(\tilde{a}), \\
& \underline{W}: \mathbb{H} \rightarrow R^{4}, \quad a \mapsto \underline{a}=\underline{W}(a) .
\end{aligned}
$$

The multiplication of two quaternions can be represented by use of these mappings as

$$
\begin{aligned}
& x y=(x y)_{0}+\widetilde{x y}, \\
& (x y)_{0}=x_{0} y_{0}-\boldsymbol{V}(\tilde{x}) \cdot \boldsymbol{V}(\tilde{y}), \\
& \widetilde{x y}=x_{0} \tilde{y}+\tilde{x} y_{0}+\boldsymbol{V}^{-1}(\boldsymbol{V}(\tilde{x}) \times \boldsymbol{V}(\tilde{y})) .
\end{aligned}
$$

Introducing the conjugate complex quaternion by

$$
x^{*}:=x_{0}-\tilde{x}
$$

we obtain for the square of its norm

$$
x x^{*}=x^{*} x=:|x|^{2}=x_{0}^{2}+\boldsymbol{V}^{2}(\tilde{x})=\underline{W}^{2}(x) \text {. }
$$

With regard to the Euler-D'Alembert proposition we now consider the special unit quaternion

$$
n=\cos (\varphi / 2)+\sin (\varphi / 2) \boldsymbol{V}^{-1}\left(\boldsymbol{n}^{0}\right) \in \mathbb{H}, \quad n^{*} n=1
$$

According to the Euler-D'Alembert proposition $0 \leq \varphi \leq \pi$ is valid, and therefore the real part of $n$ is always not negative, whereas the imaginary part depends on the

\footnotetext{
${ }^{3)}$ The letter $\mathbb{H}$ has been chosen to honour R.W. Hamilton who invented these numbers in the middle of the 19th century.
} 


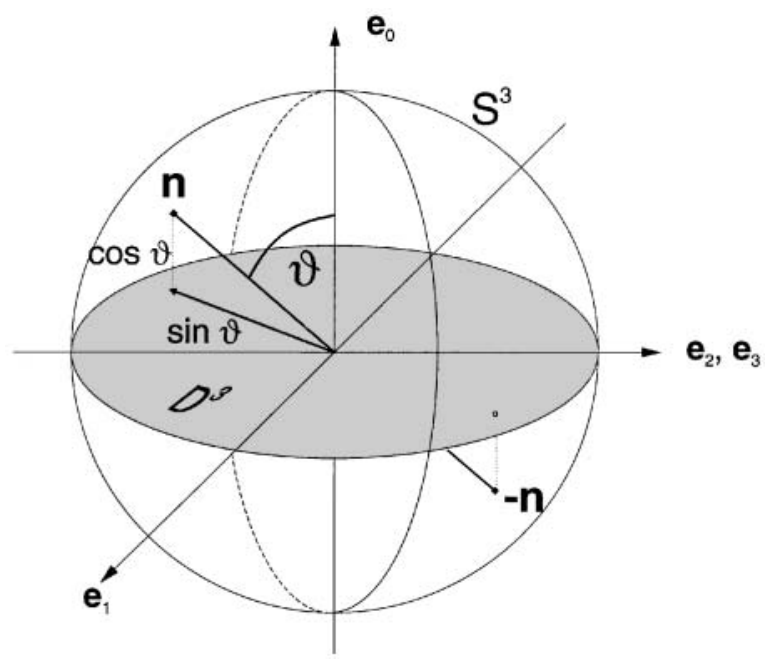

Figure 1 The antipodes $\underline{n}^{0}$ and $-\underline{n}^{0}$ of the $\mathbb{S}^{3}$ represent by projection $\Pi$ the same rotation, characterized by points of the ball $\overline{\mathbb{D}}^{3}$. Thus $\mathbb{S}^{3}$ is a universal covering of $\mathbb{D}^{3}$.

direction of $\boldsymbol{n}^{0}$. Consequently, all $n$ of negative real part do not represent a rotation, that means, not all unit 4-vectors $\underline{n}^{0}=W(n)$ decribe a rotation. This is a disadvantageous situation because the unit 4-vectors $\underline{n}^{0}=\underline{W}(n) \in \mathbb{S}^{3}$ spanning the 3dimensional sphere would be a suitable and easy set of mesoscopic variables.

The solution to this dilemma is to consider $\mathbb{S}^{3}$ as a universal covering $\mathscr{C}$ of the space spanned by the vectors belonging to the imaginary parts of $(115)$ which is the ball $\mathbb{D}^{3}$ of radius one (see Figure 1)

$$
\begin{aligned}
& \mathscr{C}=\left\langle\mathbb{S}^{3}, \mathbb{D}^{3}, \Pi\right\rangle, \quad \Pi: \mathbb{S}^{3} \rightarrow \mathbb{D}^{3}, \\
& \underline{n}^{0}:=\cos \vartheta \boldsymbol{e}_{0}+\sin \vartheta \boldsymbol{n}^{0} \mapsto \Pi\left(\underline{n}^{0}\right):=[\operatorname{sgn} \cos \vartheta] \sin \vartheta \boldsymbol{n}^{0} .
\end{aligned}
$$

This procedure is now explained in more detail. According to the Euler-D'Alembert proposition, the 3 -vector

$$
\boldsymbol{n}=\boldsymbol{V}(\tilde{n})=\sin (\varphi / 2) \boldsymbol{n}^{0} \in \mathbb{D}^{3}
$$

represents a rotation of angle $\varphi$ around the axis directed along $\boldsymbol{n}^{0}$. The imaginary part of the unit quaternion (115)

$$
\tilde{n}=\sin (\varphi / 2) \boldsymbol{V}^{-1}\left(\boldsymbol{n}^{0}\right) \in \operatorname{Im} \mathbb{H}, \quad\left|\boldsymbol{n}^{0}\right|=1
$$

is connected to this rotation by (118). Comparison of (118) with (117) makes evident that then also $\Pi\left(\underline{n}^{0}\right)$ represents a rotation. Because of

$$
\Pi\left(-\underline{n}^{0}\right)=-\{\operatorname{sgn}[-\cos (\varphi / 2)]\} \sin (\varphi / 2) \boldsymbol{n}^{0}=\Pi\left(\underline{n}^{0}\right)
$$


we obtain the result that by introducing the universal covering $\mathscr{C}(116)$, both the unit 4-vectors $n^{0}$ and $-\underline{n}^{0}$ are mapped onto the same rotation, although only one of them (that of $\cos (\varphi / 2) \geq 0$ ) is representing one. Thus two unit 4-vectors, $\underline{n}^{0} \in \mathbb{S}^{3}$, belong to each rotation.

If we now introduce $\underline{n}^{0}$ as the set of mesoscopic variables, the mesoscopic space (1) results in

$$
\left(\underline{n}^{0}, \boldsymbol{x}, t\right) \in \mathbb{S}^{3} \times \mathbb{R}^{3} \times \mathbb{R}^{1}
$$

Because $\underline{n}^{0}$ and $-\underline{n}^{0}$ belong to the same rotation, the $\operatorname{MDF}$ (2) has the head-tail symmetry (53)

$$
f\left(\underline{n}^{0}, \boldsymbol{x}, l\right)=f\left(-\underline{n}^{0}, \boldsymbol{x}, l\right) \equiv f(\bullet) .
$$

Before we can write down the local mesoscopic balances (7), we have to introduce the mesoscopic change velocity $\boldsymbol{u}(\cdot)$ defined by (8).

\subsection{Angular and mesoscopic change velocity}

Like the mesoscopic set $\underline{n}^{0}$, the mesoscopic change velocity $\boldsymbol{u}(\cdot)$ is a 4-vector defined on the mesoscopic space which indicates the change of the unit 4-vector $\underline{n}^{0}$. Therefore $\underline{u}$ is an element of the tangent space of the $\mathbb{S}^{3}$, and we have according to (111) and (113)

$$
\underline{n}^{0} \cdot \underline{u}=0=\left(n^{*} u\right)_{0} .
$$

This relation can easily be verified by introducing

$$
u \equiv \dot{n} \rightarrow \underline{u}=\underline{W}(\dot{n}),
$$

and inserting (115) into (123). According to (115) and (124) we obtain

$$
\boldsymbol{V}(\tilde{u})=(1 / 2) \cos (\varphi / 2) \dot{\varphi} \boldsymbol{n}^{0}+\sin (\varphi / 2) \dot{\boldsymbol{n}}^{0}
$$

which consists of two parts belonging to the changes in the rotation angle and the rotation axis. This 3 -vector will appear in the balance of energy. The gradient with respect to the set of mesoscopic variables now reads

$$
\nabla_{m} \cdot \boldsymbol{u} \equiv \nabla_{n} \cdot \underline{u}
$$

The angular velocity $\omega$ is defined by the time dependent proper orthogonal transformations $\mathbf{Q}(t)$ in (103)

$$
\omega:=(1 / 2) \varepsilon: \mathbf{\Omega}, \quad \mathbf{\Omega}:=\mathbf{Q}^{\top} \cdot \dot{\mathbf{Q}}
$$


Here $\varepsilon$ is the Levi-Civita tensor. Thus by introducing the tensor $\Theta$ of inertia, the angular momentum and the rotation energy are

$$
\boldsymbol{N}:=\Theta \cdot \boldsymbol{\omega}, \quad \varepsilon_{r}:=(1 / 2) \boldsymbol{\omega} \cdot \Theta \cdot \boldsymbol{\omega},
$$

and we consider the special balances for liquid crystals of biaxial molecules in the next section.

\subsection{Balances}

As we can see from (122) in comparison with (53), the argument (•) corresponds to the argument $(\cdot)$. Consequently, the balance equations written down in Section 3 are already those for biaxial liquid crystals, if we replace all arguments $(\cdot)$ by $(\bullet) \in \mathbb{S}^{3} \times \mathbb{R}^{3} \times \mathbb{R}^{1}$. The balances are now defined on the nematic space as a special case of the mesoscopic one, and some of the quantities have an other interpretation than in Section 3.

The mixed tensor $\mathscr{T}$ is the stress tensor on $\mathbb{S}^{3}$ represented by the linear mapping

$$
\mathscr{T}: \mathbb{S}^{3} \rightarrow \mathbb{R}^{3}, \quad \underline{a} \mapsto \boldsymbol{a}=\mathscr{T}(\bullet) \cdot \underline{a},
$$

$\boldsymbol{g}(\bullet)$ is the 3-vector of angular momentum exerted by the external forces on the orientation, the 3-tensor $\boldsymbol{W}(\bullet)$ and the mixed tensor

$$
\mathscr{W}: \mathbb{S}^{3} \rightarrow \mathbb{R}^{3}, \quad \underline{a} \mapsto \boldsymbol{a}=\mathscr{W}(\bullet) \cdot \underline{a},
$$

are the analogues to $\boldsymbol{T}$ and $\mathscr{T}$ and are called spin tensors, $r(\bullet)$ is the absorption supply, $\boldsymbol{q}(\bullet)$ the heat flux density, and $\boldsymbol{Q}(\bullet)$ the heat flux density on the $\mathbb{S}^{3}$.

\subsection{Alignment tensors}

According to (49) the alignment tensors are introduced as the symmetric irreducible moments of the ODF

$$
\boldsymbol{a}^{(k)}(\boldsymbol{x}, t):=\int_{S^{3}} f(\bullet) \underline{n}^{0} \ldots k \text { times } \ldots \underline{n}^{0} d^{3} n .
$$

They are macroscopic fields describing the orientational order, being non-zero in the ordered phase and vanishing in the isotropic one. Therefore they are order parameters in the sense of the theory of phase transitions. Within a purely macroscopic phenomenological theory they are internal variables independent in non-equilibrium and depending in equilibrium on the equilibrium variables density and temperature.

The equations of motion for these internal variables can be derived in the way we derived the equations of motion for the order parameters (65) in the general mesoscopic theory 


$$
\begin{aligned}
\frac{\partial}{\partial t} \underline{\boldsymbol{a}_{\ell}} & +\nabla_{x} \cdot\left[\boldsymbol{v}(\boldsymbol{x}, t) \underline{\boldsymbol{a}_{\ell}}+\int_{S^{3}}\left(\delta \boldsymbol{v}(\bullet) f(\bullet) \overline{n_{\mu_{1}}^{0} \cdots n_{\mu_{\ell}}^{0}}\right) d^{3} n\right] \\
& +\int_{S^{3}} \bar{n}_{\mu_{1}}^{0} \cdots n_{\mu_{\ell}^{0}}^{0} \nabla_{n} \cdot[\boldsymbol{u}(\bullet) f(\bullet)] d^{3} n \\
& +\underline{\boldsymbol{a}_{\ell}}\left[\frac{\partial}{\partial t} \ln \rho(\boldsymbol{x}, t)+\boldsymbol{v}(\boldsymbol{x}, t) \cdot \nabla_{x} \ln \rho(\boldsymbol{x}, t)\right]=0 .
\end{aligned}
$$

We now introduce the mesoscopic angular velocity which is different from (127)

$$
\omega(\bullet):=n \times u(\bullet) \rightarrow u(\bullet)=\omega(\bullet) \times n,
$$

and we write

$$
\boldsymbol{u}(\bullet)=\underline{\Omega}_{2}(\bullet) \cdot \boldsymbol{n},
$$

and with help of the Levi-Civita tensor we have

$$
\underline{\Omega}_{2}(\bullet):=-\varepsilon \cdot \omega(\bullet) .
$$

Using this we define the mean angular velocity tensor which is also different from (127) by

$$
\underline{\Omega}_{2}(\boldsymbol{x}, t):=\left\langle\underline{\Omega}_{2}(\bullet)\right\rangle
$$

and decompose $\underline{\Omega}_{2}(\bullet)$ according to

$$
\underline{\Omega}_{2}(\bullet):=\underline{\Omega}_{2}(\boldsymbol{x}, t)+\delta \underline{\Omega}_{2}(\bullet) .
$$

We now can prove $[53,8,20]$ the

$\square$ Proposition: The differential equation in time of the alignment tensor family is

$$
\begin{aligned}
& \frac { d } { d t } a _ { \mu _ { 1 } \cdots \mu _ { \ell } } - \ell \longdiv { \Omega _ { \mu _ { 1 } \lambda } ( \boldsymbol { x } , t ) a _ { \lambda _ { \mu _ { 2 } \cdots \mu _ { \ell } } } } \\
& =\ell\left\langle\widehat{\delta \Omega_{\mu_{1} \lambda}(\bullet) n_{\lambda}^{0} n_{\mu_{2}}^{0} \cdots n_{\mu_{\ell}}^{0}}\right\rangle-\nabla_{x} \cdot\left\langle\delta \boldsymbol{v}(\bullet) \overparen{n_{\mu_{1}}^{0} \cdots n_{\mu_{\ell}}^{0}}\right\rangle \\
& +\left\langle\delta \boldsymbol{v}(\bullet) \overline{n_{\mu_{1}}^{0} \cdots n_{\mu_{\ell}}^{0}}\right\rangle \cdot \nabla_{x} \ln \rho(\boldsymbol{x}, t) .
\end{aligned}
$$

Here are some remarks on the general structure of the equation of motion (138) of the alignment tensor family. The left hand side is Jaumanns (co-moving and corotational) time derivative of a symmetric, $\ell$-th rank tensor describing the changes of $\boldsymbol{a}_{\ell}$ which are noted by an observer travelling with velocity $\boldsymbol{v}(\boldsymbol{x}, t)$ and rotating with $\overline{\boldsymbol{\omega}}(\boldsymbol{x}, t)$. The right hand side of (138) describes the non-trivial changes of $\boldsymbol{a}_{\ell}$ that are the changes by alignment production and supply. If the peculiar velocities $\delta \boldsymbol{v}(\bullet)$ and 
$\delta \underline{\Omega}_{2}(\bullet)$ vanish, the alignment tensor $\underline{\mathbf{a}}_{\ell}$ will only change in time by the motion of the observer and not by production and supply. The right hand side of (138) contains averages with respect to $f(\bullet)$, and therefore it is a rather complicated function of all alignment tensors of the family. Thus we obtain as in (65) an infinite system of partial differential equations which does not decouple in general. The coupling is due to the moments of the peculiar velocities $\delta \boldsymbol{v}(\bullet)$ and $\delta \underline{\Omega}_{2}(\bullet)$.

\section{Summary}

The mesoscopic concept is a general tool of continuum physics for describing complex materials which are beyond hydrodynamics and thus need additional fields to represent their properties. A prominent example for a complex material are liquid crystals whose orientation of the molecules is an additional field in the meaning of the mesoscopic concept. The orientation of a single molecule, called microscopic director, is introduced as a 2- or 3-dimensional mesoscopic variable spanning the nematic space. The distribution of the microscopic directors in a volume element is described by an orientation distribution function (ODF) defined on the nematic space. Viewed historically, the mesoscopic theory of liquid crystals was developed as the last one of all the others describing liquid crystals phenomenologically. Now it appears that the mesoscopic theory can be considered as a root of all the others, because those are special cases of the mesoscopic description. Consequently, we have a family of mesoscopic theories of liquid crystals which can be characterized as follows. From the ODF we obtain on the macroscopic level of description the family of alignment tensors which was already partly introduced earlier [6] by alignment tensors of second and of fourth order. If the ODF is uniaxial, a macroscopic director field can be introduced by use of the symmetry axis of the ODF, and we obtain the so-called Maier-Saupe approach [21]. A special case of uniaxiality is the total alignment of all molecules. Here the macroscopic director field is identical to the microscopic one, and we obtain the well-known Ericksen-Leslie theory $[1,2]$ which is the most special case in the family of mesoscopic theories of liquid crystals.

\section{Acknowledgements}

The authors are grateful for financial support provided by the VISHAY Company, Selb, Germany.

\section{References}

[1] Ericksen, J.L., Anisotropic fluids, Arch. Rat. Mech. Anal., 4 (1960), 231-237.

[2] Leslie, F.M., Some constitutive equations for liquid crystals, Arch. Rat. Mech. Anal., 28 (1968), 265-283.

[3] Muschik, W., Ehrentraut, H., Blenk, S., Ericksen-Leslie liquid crystal theory revisited from a mesoscopic point of view, J. Non-Equilib. Thermodyn., 20 (1995), 92-101.

[4] Eringen, A.C., Lee, J.D., Relations of two continuum theories of liquid crystals, in: Liquid Crystals and Ordered Fluids, eds. J.F. Johnson, R.S. Porter, vol. 2, pp. 315-330, Plenum Press, New York, 1974. 
[5] Eringen, A.C., Micropolar theory of liquid crystals, in: Liquid Crystals and Ordered Fluids, eds. J.F. Johnson, R.S. Porter, vol. 3, pp. 443-474, Plenum Press, New York, 1978.

[6] Hess, S., Irreversible thermodynamics of nonequilibrium alignment phenomena in molecular liquids and in liquid crystals, Z. Naturforsch., 30a (1975), 728-733.

[7] Pardowitz, I., Hess, S., On the theory of irreversible processes in molecular liquids and liquid crystals, nonequilibrium phenomena associated with the second and fourth rank alignment-tensors, Physica A, 100 (1980), 540-562.

[8] Ehrentraut, H., Muschik, W., On symmetric irreducible tensors in d-dimensions, ARI, 51 (1998), 149-159.

[9] Muschik, W., Ehrentraut, H., Papenfuss, C., Blenk, S., Mesoscopic theory of liquid crystals, in: 25 Years of Non-Equilibrium Statistical Mechanics, Proceedings of the XIII Sitges Conference, June 13-17, 1994, Sitges, Spain, eds. J.J. Brey, J. Marro, J.M. Rubi, M. San Miguel, Lecture Notes in Physics, pp. 303-311, Springer, Berlin, 1995.

[10] Blenk, S., Eine statistische Begründung phänomenologischer Bilanzgleichungen für Flüssigkristalle im Rahmen einer mikroskopischen Direktortheorie, Thesis, Institut für Theoretische Physik, Technische Universität Berlin, 1989.

[11] Blenk, S., Muschik, W., Orientational balances for nematic liquid crystals, J. NonEquilib. Thermodyn., 16 (1991), 67-87.

[12] Blenk, S., Ehrentraut, H., Muschik, W., Statistical foundation of macroscopic balances for liquid crystals in alignment tensor formulation, Physica A, 174 (1991), 119-138.

[13] Blenk, S., Ehrentraut, H., Muschik, W., A continuum theory for liquid crystals describing different degrees of orientational order, Liquid Crystals, 14 (1993), 1221-1226.

[14] Müller, W.H., Muschik, W., Bilanzgleichungen offener mehrkomponentiger Systeme I, Massen- und Impulsbilanzen, J. Non-Equilib. Thermodyn., 8 (1983), 29-46.

[15] Muschik, W., Müller, W.H., Bilanzgleichungen offener mehrkomponentiger Systeme II, Energie- und Entropiebilanz, J. Non-Equilib. Thermodyn., 8 (1983), 47-66.

[16] Ehrentraut, H., Muschik, W., Balance laws and constitutive equations of microscopic rigid bodies: A model for biaxial liquid crystals, Mol. Cryst. Liq. Cryst., 262 (1995), 561568.

[17] Papenfuss, C., Muschik, W., Constitutive theory for two-dimensional liquid crystals, Mol. Cryst. Liq. Cryst., 262 (1995), 473-484.

[18] Papenfuss, C., Muschik, W., Continuum thermodynamics of two-dimensional liquid crystals, Extracta Mathematicae, 11 (1996), 181-191.

[19] Papenfuss, C., Muschik, W., Theory of two-dimensional liquid crystals - Influence of curvature, liquid cristalline ordering and electromagnetic fields, Thin Solid Films, 284285 (1996), 484-488.

[20] Muschik, W., Ehrentraut, H., Papenfuss, C., Concepts of mesoscopic continuum physics with application to biaxial liquid crystals, J. Non-Equilib. Thermodyn., 2000 (25), 179197.

[21] Maier, W., Saupe, A., Eine einfache molekular-statistische Theorie der nematischen kristallinflüssigen Phase, Teil 1, Z. Naturforsch. A, 14 (1959), 882-889.

[22] Frank, F.C., Liquid crystals, On the theory of liquid crystals, Discussion Faraday Soc., 25 (1958), 19-28.

[23] Ericksen, J.L., Conservation laws for liquid crystals, Trans. Soc. Rheol., 5 (1961), 23-34.

[24] Ericksen, J.L., Hydrostatic theory of liquid crystals, Arch. Rat. Mech. Anal., 9 (1962), 371-378.

[25] Hess, S., Waldmann, L., Kinetic theory for a dilute gas of particles with spin, Z. Naturforsh. A, 21 (1966), 1529-1546.

[26] Hirning, R., Funk, W., Trebin, H.-R., Schmidt, M., Schmiedel, H., Threshold behaviour and electro-optical properties of twisted nematic layers with weak anchoring in the tild and twist angle, J. Appl. Physics, 70 (1991), 4211-4216.

[27] Virga, E.G., Variational Theories for Liquid Crystals, Section 1.3.7, Applied Mathematics and Mathematical Computation 8, Chapman \& Hall, London, 1994.

[28] Roccato, D., Virga, E.G., On surface defects in nematic liquid crystals with variable degree of orientation, Publicatione di Università di Pisa, 4 (1991), 1-25. 
[29] Kitzerow, H.-S., Polymer-dispersed liquid crystals. From the nematic curvilinear aligned phase to ferroelectric films, Liquid Crystals, 16 (1994), 1-31.

[30] Ericksen, J.L., Liquid crystals with variable degree of orientation, Arch. Rat. Mech. Anal., 113 (1991), 97-120.

[31] Muschik, W., Papenfuss, C., Ehrentraut, H., A sketch of continuum thermodynamics, J. Non-Newtonian Fluid Mech., 96 (2001), 255-290, section 5.

[32] Muschik, W., Papenfuss, C., Ehrentraut, H., Alignment tensor dynamics induced by the mesoscopic balance of the orientation distribution function, Proc. Estonian Acad. Sci. Phys. Math., 46 (1997), 94-101.

[33] Papenfuss, C., Muschik, W., Orientational order in free standing liquid crystalline films and derivation of a closure relation for higher order alignment tensors, Mol. Cryst. Liq. Cryst., 330 (1999), 541-548.

[34] Papenfuss, C., Nonlinear dynamics of the alignment tensor in the presence of electric fields, Arch. Mech., 50 (1998), 529-536.

[35] Ehrentraut, H., Muschik, W., Papenfuss, C., Mesoscopically derived orientation dynamics of liquid crystals, J. Non-Equilib. Thermodyn., 22 (1997), 285-298.

[36] Hess, S., Fokker-Planck-Equation approach to flow alignment in liquid crystals, Z. Naturforsch. A, 31 (1976), 1034-1037.

[37] Vertogen, G., de Jeu, W.H., Thermotropic Liquid Crystals, Fundamentals, sect. 1.1, Springer Series in Chemical Physics 45, Berlin, Springer, 1988.

[38] Landau, L.D., Collected Papers, ed. D. Ter Haar, Gordon and Breach, New York, 1981.

[39] Longa, L., Monselesan, D., Trebin, H.-R., An extension of the Landau-Ginzburg-de Gennes theory for liquid crystals, Liquid Crystals, 2 (1987), 769-796.

[40] Doi, M., Larson, R., The effect of steady flow fields on the isotropic-nematic phase transition of rigid rod-like polymers, J. Chem. Phys., 92 (1990), 792-800.

[41] Hess, S., Irreversible thermodynamics of nonequilibrium alignment phenomena in molecular liquids and in liquid crystals II, Z. Naturforsch. A, 30 (1975), 1224-1232.

[42] R. Courant, D. Hilbert, Methods of Mathematical Physics, vol. 1, pp. 2 and 49, New York, Interscience 1953.

[43] Leslie, F.J., Some constitutive equations for liquid crystals, Arch. Rat. Mech. Anal., 28 (1965), 265.

[44] De Gennes, P.G., The Physics of Liquid Crystals, p. 24, Oxford University Press, Oxford, 1974.

[45] Blenk, S., Ehrentraut, H., Muschik, W., Macroscopic constitutive equations for liquid crystals induced by their mesoscopic orientation distribution, Int. J. Engng. Sci., 30 $\overline{(1992)}, 1 \overline{127-1143 .}$

[46] de Gennes, P.G., The Physics of Liquid Crystals, Chap. 3, Oxford University Press, Oxford, 1974.

[47] Kelker, H., Hatz, R., Handbook of Liquid Crystals, Verlag Chemie, Weinheim, 1980.

[48] Ehrentraut, H., personal communication, 1995.

[49] Ehrentraut, H., Hess, S., Viscosity coefficients of partially aligned nematic and nematic discotic liquid crystals, Phys. Rev. E, 51 (1995), 2203-2212.

[50] Baals, D., Hess, S., The viscosity coefficients of oriented nematic and nematic discotic liquid crystals; Affine transformation model, Z. Naturforsch. A, 43 (1988), 662-670.

[51] Jacobsen, E.A., Swift, J., Hydrodynamics of biaxial nematic liquid crystals, Mol. Cryst. Liq. Cryst., 78 (1981), 311-317.

[52] Carlsson, T., Leslie, F.M., Behaviour of biaxial nematics in the presence of electric and magnetic fields, Liquid Crystals, 10 (1991), 325-340.

[53] Ehrentraut, H., A Unified Mesoscopic Continuum Theory of Uniaxial and Biaxial Liquid Crystals, Wissenschaft- und Technik Verlag, Berlin, 1996.

[54] Harauz, G., Representation of rotations by unit quaternions, Ultramicroscopy, 33 (1990), 209-213.

Paper received: 2003-04-22

Paper accepted: 2003-06-02

J. Non-Equilib. Thermodyn. · $2004 \cdot$ Vol. $29 \cdot$ No. 1 